Review

\title{
Mechanisms of Suppression and Enhancement of Photocurrent/Conversion Efficiency in Dye-Sensitized Solar-Cells Using Carotenoid and Chlorophyll Derivatives as Sensitizers
}

\author{
Yasushi Koyama $^{1, *}$, Yoshinori Kakitani ${ }^{1}$ and Hiroyoshi Nagae ${ }^{2}$ \\ 1 Faculty of Science and Technology, Kwansei Gakuin University, 2-1 Gakuen, \\ Sanda 669-1337, Japan \\ 2 Kobe City University of Foreign Studies, 9-1 Gakuen-Higashimachi, Nishi-ku, \\ Kobe 651-2187, Japan
}

* Author to whom correspondence should be addressed; E-Mail: ykoyama@kwansei.ac.jp; Tel./Fax: +81-79-559-6577.

Received: 10 December 2011; in revised form: 14 February 2012 / Accepted: 15 February 2012 / Published: 22 February 2012

\begin{abstract}
The mechanisms of suppression and enhancement of photocurrent/conversion efficiency (performance) in dye-sensitized solar cells, using carotenoid and chlorophyll derivatives as sensitizers, were compared systematically. The key factor to enhance the performance was found to be how to minimize interaction among the excited-state dye-sensitizer(s). In a set of retinoic-acid (RA) and carotenoic-acid (CA) sensitizers, having $n$ conjugated double bonds, CA7 gave rise to the highest performance, which was reduced toward RA5 and CA13. The former was ascribed to the generation of triplet and the resultant singlet-triplet annihilation reaction, while the latter, to the intrinsic electron injection efficiency. In a set of shorter polyene sensitizers having different polarizabilities, the one with the highest polarizability (the highest trend of aggregate formation) exhibited the higher performance toward the lower dye concentration and the lower light intensity, contrary to our expectation. This is ascribed to a decrease in the singlet-triplet annihilation reaction. The performance of cosensitization, by a pair of pheophorbide sensitizers without and with the central metal, $\mathrm{Mg}$ or $\mathrm{Zn}$, was enhanced by the light absorption (complementary rather than competitive), the transition-dipole moments (orthogonal rather than parallel) and by the pathways of electron injection (energetically independent rather than interactive).
\end{abstract}


Keywords: dye-sensitized solar cells; carotenoid and chlorophyll derivatives; singlet-triplet annihilation

\section{Nomenclature}

Some key concepts for those readers who are non-specialized in physical chemistry:

RA and CAs: RA (retinoic acid) and CA (carotenoic acid) are just the traditional classification depending on the conjugation length. RA5 can be written as CA5, as well.

$I-V$ curve: The correlation between $I$ (photocurrent) and $V$ (photovoltage) characterizes the performance of a solar cell. When $V=0$, the photocurrent flux is called $J_{\text {sc }}$ (short-circuit photocurrent flux), whereas when $J_{\mathrm{sc}}=0$, the photovoltage is called $V_{\mathrm{oc}}$ (open-circuit photovoltage). The area of a rectangle surrounded by the $I-V$ curve reflects $\eta$ (solar energy-to-electricity conversion efficiency).

SVD (singular-value decomposition) and global-fitting: A method of spectral analysis to reconstitute the observed time-resolved difference spectra as SADS (species-associated difference spectra) (generated species positive, lost species negative) multiplied by time-dependent changes in population, and take a sum of them at each delay time. Perfect fitting can be obtained only when the kinetic model (the time constants of the relevant components) is (are) completely correct. Continuous fluctuation (noise) can be removed during these analytical processes.

CBE (conduction-band-edge): The lowest edge of the conduction band of the semiconductor to which electron can be injected into from the excited state of a dye-sensitizer.

IPCE (incident photon-to-current conversion efficiency): The efficiency of conversion, at each wavelength, from the incident photon to electron (\%).

HOMO (the highest-occupied molecular orbital) and LUMO (the lowest-unoccupied molecular orbital): In linear or circular conjugated chains, a staircase-type set of electronic levels is formed in the order ... LUMO+1 $>$ LUMO $>$ HOMO $>$ HOMO-1 ... symmetric with a line just in-between LUMO and HOMO.

\section{Conjugation-Length Dependence of Excited-State Dynamics Affecting Photocurrent/ Conversion Efficiency in Retinoic-Acid and Carotenoic-Acid Sensitizers}

Polyenes have a linear conjugated system, from which electrons can be injected into $\mathrm{TiO}_{2}$ when a carboxyl group is attached to facilitate binding and electron injection. As a set of sensitizers, we used a retinoic acid (RA) and carotenoic acids (CAs) having $n=5-13$ double bonds (Figure 1). The dependence of their excited-state energetics and dynamics on the conjugation length $(n)$ has been well-documented [1,2].

We first examined the conjugation-length dependence of the photocurrent and conversion efficiency (collectively called 'performance') of solar cells using the set of sensitizers, and tried to explain the results in terms of the excited-state dynamics of RA and CAs free in solution and bound to $\mathrm{TiO}_{2}$ nanoparticles in suspension. The highest performance was obtained with CA7; its decline toward CA13 was explained by the electron-injection efficiency, whereas that seen toward RA5 was explained partially in terms of triplet generation at later stages after excitation, as will be described below: 
Figure 1. Chemical structures of retinoic acid (RA5) and carotenoic acids (CA6-CA13) having $n=5-13$ conjugated double bonds.

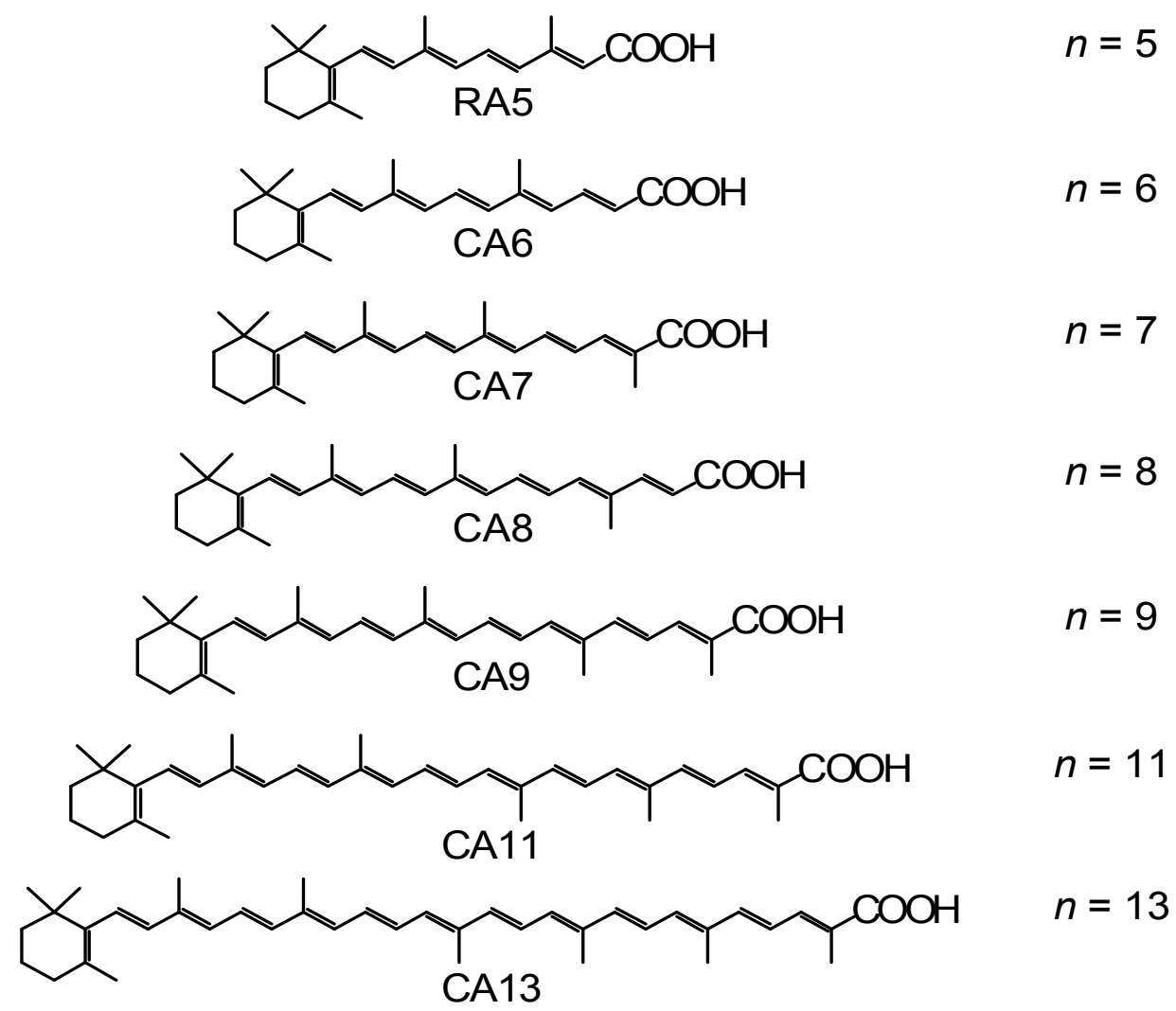

Figure 2 shows the $I-V$ curves of solar cells using the set of sensitizers [3]. The short-circuit photocurrent density $\left(J_{\text {sc }}\right)$ is in the order, RA5 $<$ CA6 $<$ CA7 $>$ CA $8>$ CA9 $>$ CA11 $>$ CA13, whereas the open-circuit photovoltage $\left(V_{\text {oc }}\right)$ is in the order, RA5 $>$ CA6 $>$ CA7 $>$ CA8; CA8, CA9, CA11 and CA13 are exhibiting similar values.

Figure 2. Conjugation-length $(n)$ dependence of the $I-V$ curves in solar cells using RA and CA sensitizers (reprinted from [3] with permission from Elsevier).

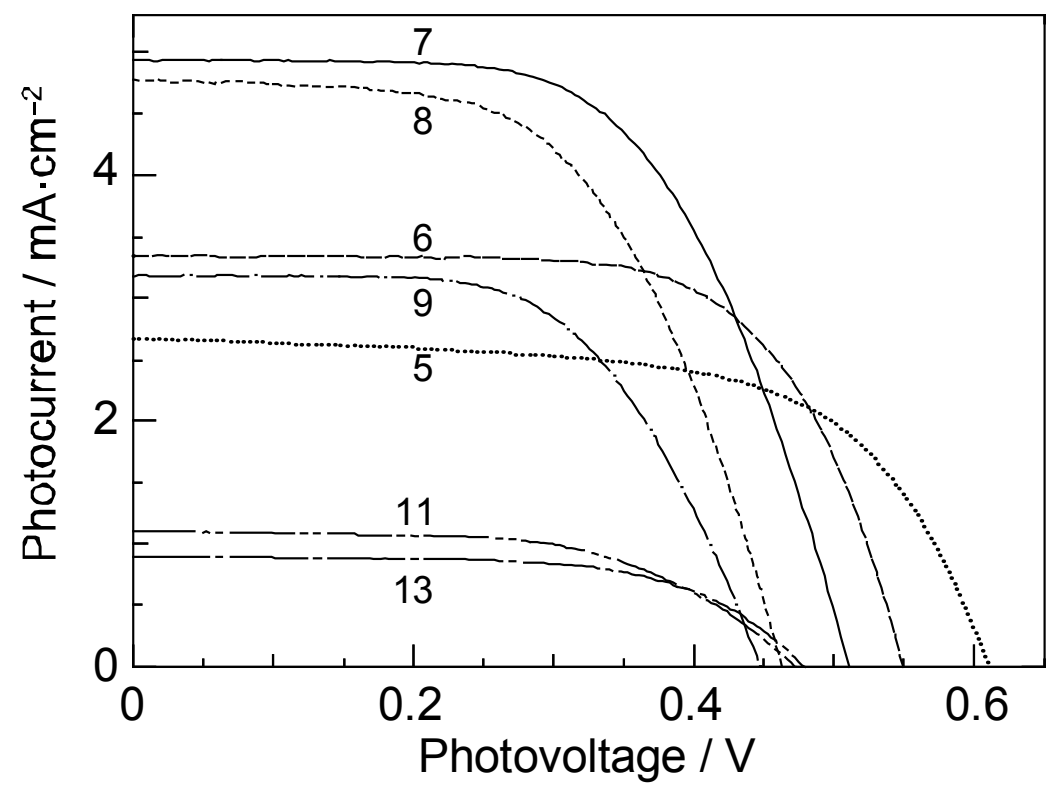


Presumably, the coverage on the surface of $\mathrm{TiO}_{2}$ layer should be better-organized in the shorterchain RA5, CA6 and CA7 sensitizers in the complete all-trans configuration; the longer-chain sensitizers tend to form $c i s$ isomers, as well. Open-circuit photovoltage $\left(V_{\mathrm{oc}}\right)$ must reflect this situation. Figure $3 \mathrm{a}, \mathrm{b}$ presents the conjugation-length dependence of short-circuit current density $\left(J_{\mathrm{sc}}\right.$, hereafter simply called 'photocurrent') and the solar energy-to-electricity conversion efficiency ( $\eta$, called 'conversion efficiency') [3].

Figure 3. Conjugation-length $(n)$ dependence of (a) the photocurrent $\left(J_{\mathrm{sc}}\right)$ and $(\mathbf{b})$ the conversion efficiency $(\eta)$ in solar cells using the RA and CA sensitizers, and (c) the electron-injection efficiency $(\Phi)$ in the RA and CA sensitizers bound to $\mathrm{TiO}_{2}$ nanoparticles in suspension (reprinted from [4] with permission of the American Chemical Society).

(a)

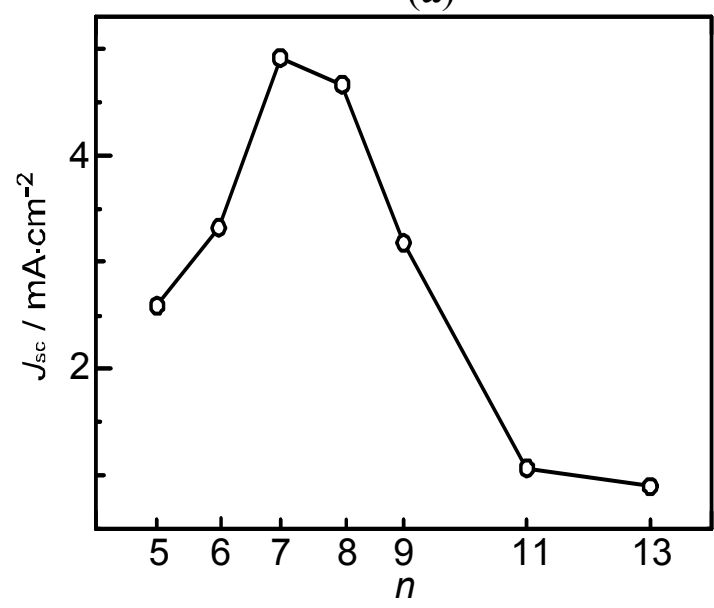

(b)

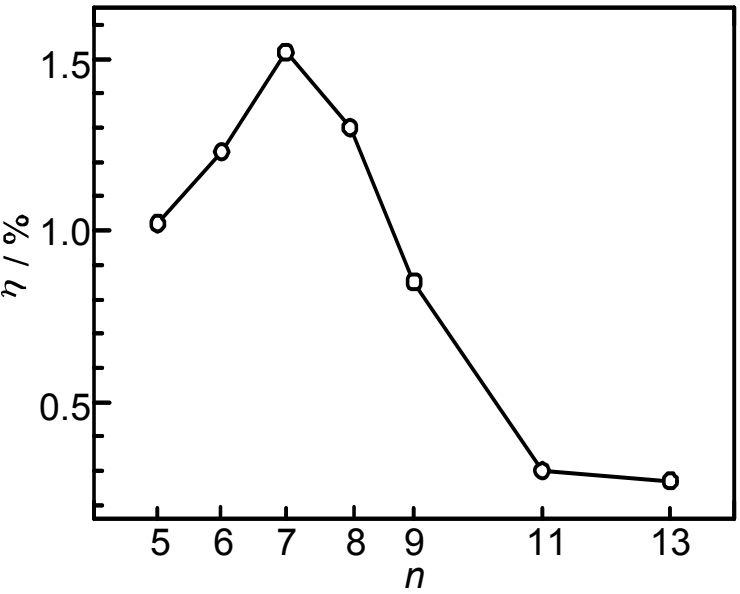

(c)

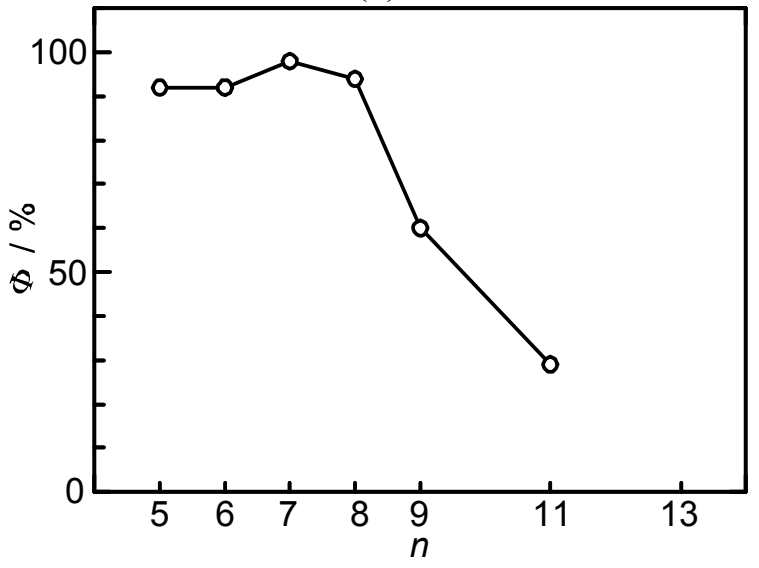

The performance is at the maximum in CA7; they decline toward the shorter chain, in the order, CA6 and RA5, while toward the longer chain, in the order, CA8, CA9, CA11 and CA13, reflecting the trends of $J_{\text {sc }}$ and $V_{\text {oc }}$ mentioned above.

To understand the mechanisms giving rise to the above dependence of photocurrent and conversion efficiency on $n$, we examined the excited-state dynamics of the set of sensitizers: Figure 4 shows an energy diagram for the $\pi$-conjugated chains of RA and CAs with $n=5-13$ [4]: the linear dependence of the optically-active $1 \mathrm{~B}_{\mathrm{u}}^{+}$state, as a function of $1 /(2 n+1)$, was determined by conventional electronic-absorption spectroscopy. 
Figure 4. An energy diagram for the optically-allowed $1 \mathrm{~B}_{\mathrm{u}}{ }^{+}$and optically-forbidden $2 \mathrm{~A}_{\mathrm{g}}{ }_{\mathrm{e}}$, $1 \mathrm{~B}_{\mathrm{u}}{ }^{-}$and $3 \mathrm{Ag}_{\mathrm{g}}{ }^{-}$states for RA an CAs having $n=5-13$ conjugated double bonds (reprinted from [4] with permission of the American Chemical Society).

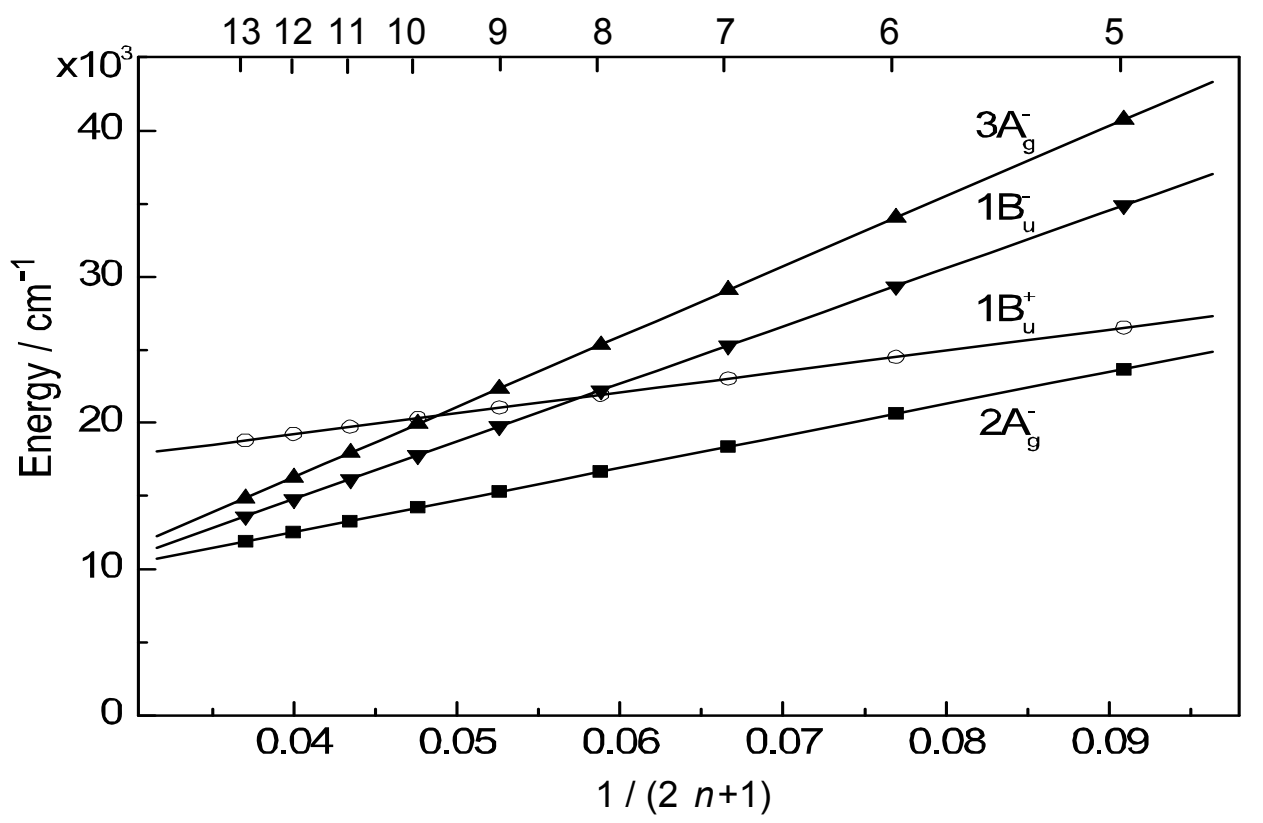

The linear dependences of the optically-forbidden $1 \mathrm{~B}_{\mathrm{u}}{ }^{-}, 3 \mathrm{~A}_{\mathrm{g}}{ }^{-}$and $2 \mathrm{Ag}_{\mathrm{g}}{ }^{-}$states are transferred from those of bacterial Cars $(n=9-13)$ determined by the measurement of resonance-Raman excitation profiles [5]; the energies for CA8-RA5 were the extrapolation of these linear relations. According to the state ordering, after excitation to the $1 \mathrm{~B}_{\mathrm{u}}{ }^{+}$state by the absorption of photon, (i) RA5, CA6, CA7 and CA8 are expected to internally convert, in the order, $1 \mathrm{~B}_{\mathrm{u}}^{+} \rightarrow 2 \mathrm{~A}_{\mathrm{g}}{ }^{+} \rightarrow 1 \mathrm{~A}_{\mathrm{g}}{ }^{-}$(the ground state), (ii) CA9 and $\mathrm{CA} 10$, in the order, $1 \mathrm{~B}_{\mathrm{u}}^{+} \rightarrow 1 \mathrm{~B}_{\mathrm{u}}^{-} \rightarrow 2 \mathrm{~A}_{\mathrm{g}}{ }^{-} \rightarrow 1 \mathrm{~A}_{\mathrm{g}}{ }^{-}$and (iii) $\mathrm{CA} 11$, in the order, $1 \mathrm{~B}_{\mathrm{u}}^{+} \rightarrow 3 \mathrm{~A}_{\mathrm{g}}{ }^{-} \rightarrow 1 \mathrm{~B}_{\mathrm{u}}^{-} \rightarrow 2 \mathrm{Ag}^{-} \rightarrow 1 \mathrm{~A}_{\mathrm{g}}{ }^{-}$as expected by the energy diagram.

On the basis of the above set of energy levels and internal conversion processes, we analyzed, by means of singular-value-decomposition (SVD) followed by global fitting, the time-resolved data matrices, for the set of RA5-CA11 sensitizers free in solution and bound to $\mathrm{TiO}_{2}$ nanoparticles in suspension.

Figure 5 presents the internal-conversion and electron-injection pathways and the relevant time constants for the free and bound states [4], which will be characterized below:

We start with the cases of RA and CAs free in solution: In RA 5, rapid transformation from the $1 \mathrm{~B}_{\mathrm{u}}{ }^{+}$to the $2 \mathrm{~A}_{\mathrm{g}}{ }^{-}$state followed by the generation of radical cation $\left(\mathrm{D}_{0}{ }^{\cdot+}\right)$ was observed. In CA6-CA8, rapid $1 \mathrm{~B}_{\mathrm{u}}{ }^{+} \rightarrow 2 \mathrm{~A}_{\mathrm{g}}{ }^{-}$transformation followed by the slow decay of the $2 \mathrm{~A}_{\mathrm{g}}{ }^{-}$state was observed; here, no generation of $\mathrm{D}_{0}{ }^{++}$was seen. In CA9 and CA11, direct transformation from the $1 \mathrm{~B}_{\mathrm{u}}{ }^{+}$to the $2 \mathrm{~A}_{\mathrm{g}}{ }^{-}$state was not seen in the visible region, but rapid transformation from the $1 \mathrm{~B}_{\mathrm{u}}{ }^{+}$to the $1 \mathrm{~B}_{\mathrm{u}}{ }^{-}$state and that from the $1 \mathrm{~B}_{\mathrm{u}}^{+}$to the $3 \mathrm{~A}_{\mathrm{g}}{ }^{-}$state, respectively, were seen in the near-infrared region. Their spectral patterns agreed with those of the $1 \mathrm{~B}_{\mathrm{u}}{ }^{-}$and $3 \mathrm{~A}_{\mathrm{g}}{ }^{-}$states of carotenoids, i.e., neurosporene $(n=9)$ and lycopene $(n=11)$, respectively [6]. The time-dependent changes in population in CA9 showed extremely-rapid $1 \mathrm{~B}_{\mathrm{u}}^{+} \rightarrow 1 \mathrm{~B}_{\mathrm{u}}^{-}$transformation followed by the slower $1 \mathrm{~B}_{\mathrm{u}}^{-} \rightarrow 2 \mathrm{~A}_{\mathrm{g}}{ }^{-}$transformation, whereas those in CA11, extremely-rapid $1 \mathrm{~B}_{\mathrm{u}}{ }^{+} \rightarrow 3 \mathrm{~A}_{\mathrm{g}}{ }^{-}$transformation followed by the slower $3 \mathrm{Ag}_{\mathrm{g}}{ }^{-} \rightarrow$ $2 \mathrm{~A}_{\mathrm{g}}{ }^{-}$transformation $\left(1 \mathrm{~B}_{\mathrm{u}}^{-}\right.$was skipped). Both were followed by the $2 \mathrm{~A}_{\mathrm{g}}{ }^{-} \rightarrow 1 \mathrm{~A}_{\mathrm{g}}{ }^{-}$transformation. 
Figure 5. The pathways of internal conversion and electron injection for the RA and CA sensitizers free in solution and bound to $\mathrm{TiO}_{2}$ nanoparticles in suspension. The time constant for each pathway is shown in picoseconds (the $T_{1}$ state generated together with the $\mathrm{D}_{0}{ }^{++}$state when bound which is not indicated) (reprinted from [4] with permission of the American Chemical Society).
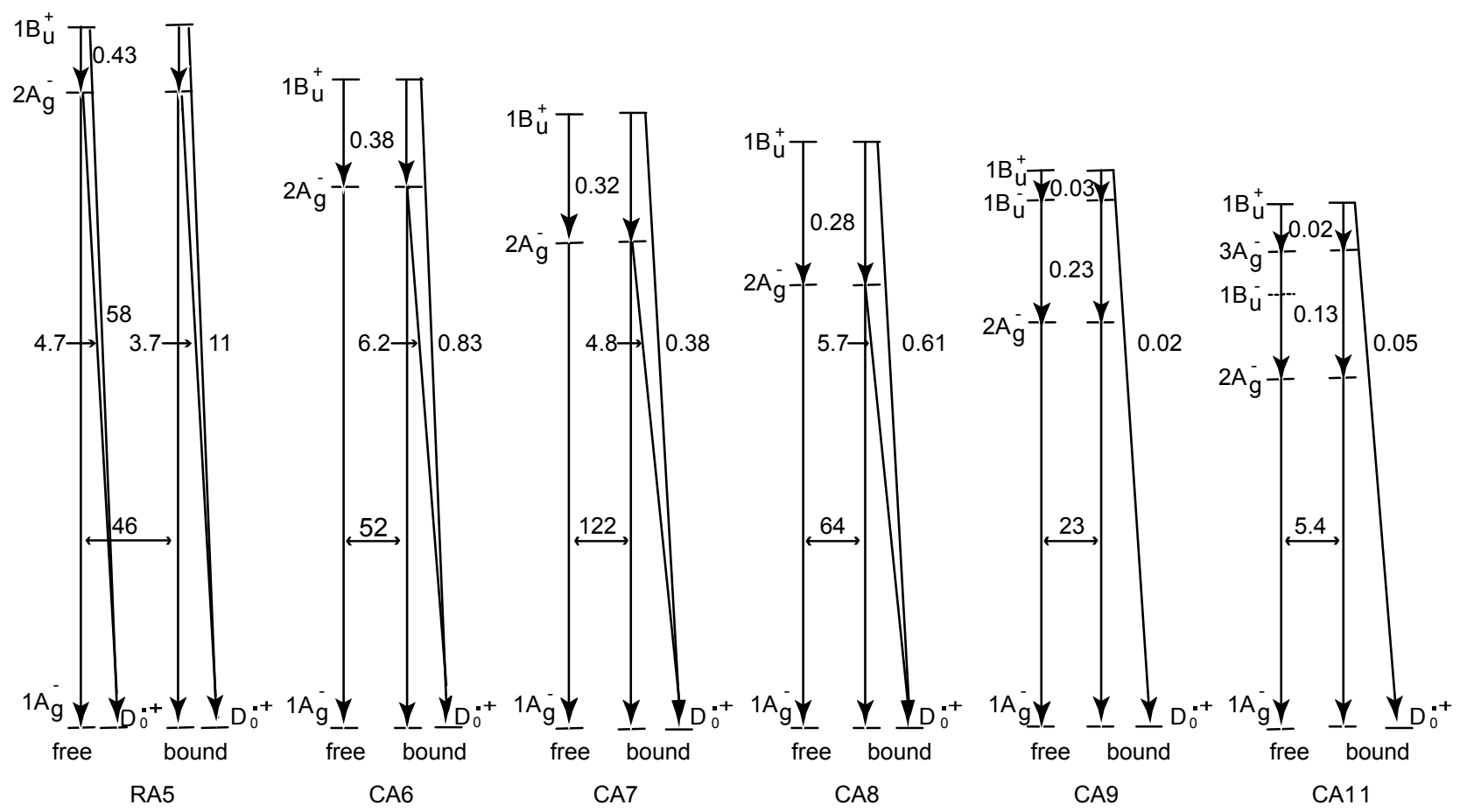

Then, we proceed to the cases of RA5-CA11 bound to $\mathrm{TiO}_{2}$ nanoparticles in suspension: the singlet-excited states generated by the photo-excitation of the sensitizers bound to $\mathrm{TiO}_{2}$ were basically the same as those generated free in solution. The most conspicuous difference in the excited-state dynamics, in the bound state, is that the transient absorptions of the triplet $\left(\mathrm{T}_{1}\right)$ and the radical-cation $\left(\mathrm{D}_{0}{ }^{-+}\right)$states appears immediately after electron injection (data not shown). The former transient absorptions agree, in energy, with those of the $T_{1}$ states obtained by anthracene-sensitized photoexcitation, whereas the latter transient absorptions, with the stationary-state absorptions of radical cation obtained electrochemically, both free in solution. The generation of the apparent $\mathrm{D}_{0}{ }^{*+}+\mathrm{T}_{1}$ state, however, drastically influences the dynamics of singlet-excited states (note that only the $\mathrm{D}_{0}{ }^{*+}$ state is shown in Figure 5). In RA5-CA8, the generation of the $\mathrm{D}_{0}{ }^{\cdot+}+\mathrm{T}_{1}$ state substantially accelerates the decay of both the $1 \mathrm{~B}_{\mathrm{u}}^{+}$and $2 \mathrm{~A}_{\mathrm{g}}{ }^{-}$states, showing efficient electron injection from these excited states into $\mathrm{TiO}_{2}$. In CA9 and CA11, on the other hand, it accelerates the decay of not the $2 \mathrm{~A}_{\mathrm{g}}{ }^{-}$state but the $1 \mathrm{~B}_{\mathrm{u}}^{+}$state, showing that electron injection was taking place only from the latter. This apparent $\mathrm{D}_{0}{ }^{++}+\mathrm{T}_{1}$ state stayed for $10 \mathrm{ps}$ in RA5-CA8 and for $1.0 \mathrm{ps}$ in CA9 and CA11, as far as the time range of pump-probe electronic-absorption spectroscopy.

Table 1 lists the electron-injection efficiencies through the $1 \mathrm{~B}_{\mathrm{u}}{ }^{+}$and $2 \mathrm{~A}_{\mathrm{g}}{ }^{-}$channels and a sum of the two, for the set of RA and CAs [7], which were calculated by the use of those time constants. The conjugation-length dependence of the total electron-injection efficiency $(\Phi)$ is depicted in Figure $3 c$. The highest efficiency was seen in CA7 (almost unity); the decline toward the longer-chain, i.e., CA7 $>$ CA8 $>$ CA9 $>$ CA11, reflects the intrinsic excited-state dynamics of the Car conjugated chain. 
However, the decline toward CA6 and RA5 is left unexplained. Table 2 shows that the values of one electron-oxidation potential systematically lowers with $n$ [7], a trend which predicts the electroninjection efficiency monotonically increasing with $n$, all the way from $n=5$ to 11 , contrary to the observation in the fabricated solar cells.

Table 1. Electron-injection efficiencies through the $1 \mathrm{~B}_{\mathrm{u}}{ }^{+}$and the $2 \mathrm{~A}_{\mathrm{g}}{ }^{-}$channels and a sum of them, calculated by the use of time constants shown in Figure 5 (reprinted from [7] with permission of MDPI Publishing).

\begin{tabular}{ccccccc}
\hline & RA5 & CA6 & CA7 & CA8 & CA9 & CA11 \\
\hline $\mathbf{1 B}_{\mathbf{u}}{ }^{+}$channel & 0.04 & 0.31 & 0.46 & 0.31 & 0.60 & 0.29 \\
$\mathbf{2 A}_{\mathbf{g}}{ }^{-}$channel & 0.88 & 0.61 & 0.52 & 0.63 & - & - \\
Sum & 0.92 & 0.92 & 0.98 & 0.94 & 0.60 & 0.29 \\
\hline
\end{tabular}

Table 2. One-electron oxidation potentials in dichloromethane (in V) (reprinted from [7] with permission of MDPI Publishing).

\begin{tabular}{lcccccc}
\hline & RA5 & CA6 & CA7 & CA8 & CA9 & CA11 \\
\hline $\boldsymbol{E}_{\text {ox }}($ vs. $\mathbf{A g} / \mathbf{A g C l})$ & 1.08 & 0.97 & 0.87 & 0.80 & 0.77 & 0.71 \\
\hline
\end{tabular}

We have observed the generation of 'the $\mathrm{D}_{0}{ }^{++}+\mathrm{T}_{1}$ state' just by transient absorptions, which does not decay at all in the time scales mentioned above. Therefore, we do not know, at this moment, what we now call 'the $\mathrm{D}_{0}{ }^{\cdot+}+\mathrm{T}_{1}$ state' is either 'a combined $\mathrm{D}_{0}{ }^{\cdot+}+\mathrm{T}_{1}$ state' or 'a mixture of the $\mathrm{D}_{0}{ }^{\cdot+}$ state and the $T_{1}$ state'. We have applied submicrosecond pump-probe spectroscopy to examine the later stages after excitation. Therefore, we focused our attention on the later time region.

Figure 6 shows the results of the analysis of submicrosecond time-resolved data for the four shorterchain RA and CAs [4]. Here, a relaxation mechanism, including the splitting of a combined $\mathrm{D}_{0}{ }^{*+}+\mathrm{T}_{1}$ state into a pair of the $\mathrm{D}_{0}{ }^{++}$and $\mathrm{T}_{1}$ states, has been nicely explained. The first spectral patterns (upper panels) show that the $\mathrm{T}_{1} / \mathrm{D}_{0}{ }^{\cdot+}$ population ratio in the combined $\mathrm{D}_{0}{ }^{\cdot+}+\mathrm{T}_{1}$ state increases toward RA5. Consistently, the time-dependent changes in population (lower panels) show that the ratio of the split $\mathrm{T}_{1} / \mathrm{D}_{0}{ }^{++}$species also increases toward RA5.

Figure 6. SADS (upper panels) and time-dependent changes in population (lower panels), obtained by singular-value decomposition (SVD) followed by global fitting, for RA5-CA8 bound to $\mathrm{TiO}_{2}$ nanoparticles in suspension (reprinted from [4] with permission of the American Chemical Society).
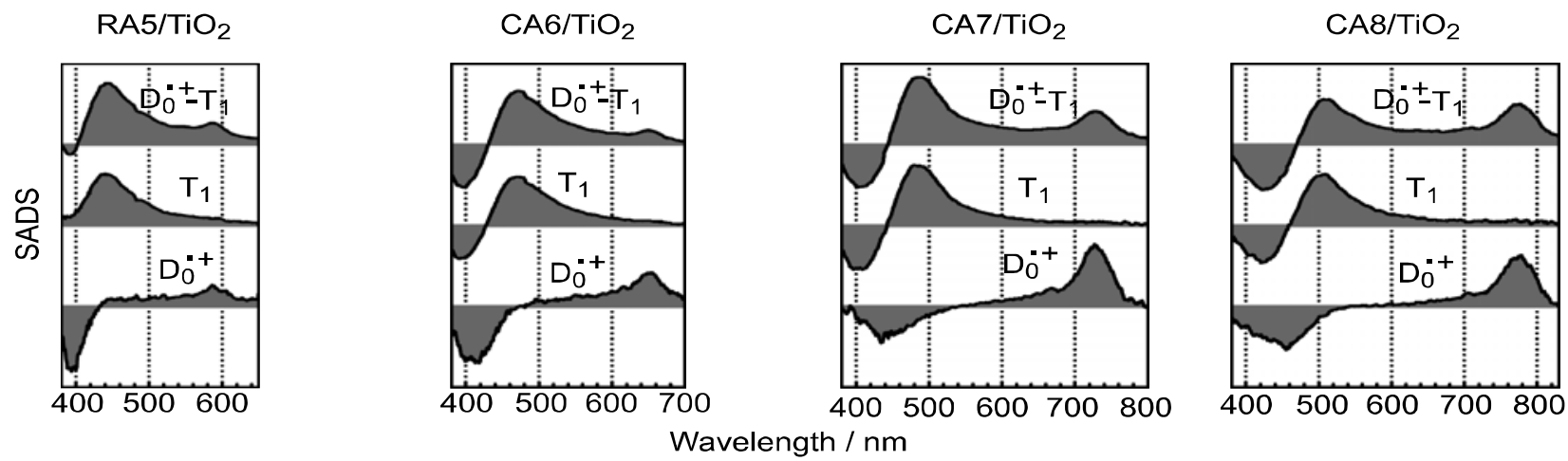
Figure 6. Cont.
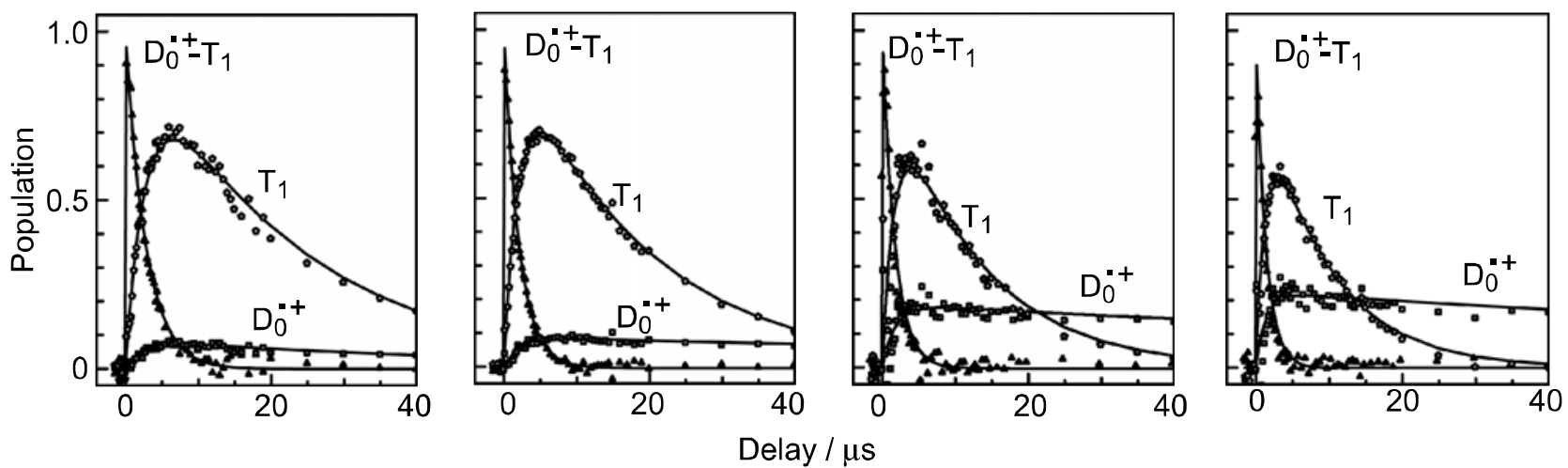

Table 3 lists the quantum yields for the $\mathrm{D}_{0}{ }^{\cdot+}$ and $\mathrm{T}_{1}$ species $\left(\phi_{\mathrm{D}}\right.$ and $\phi_{\mathrm{T}}$ ) calculated by the use of the relevant time constants in Figure 5 [4]. The efficiency of electron injection $\left(\phi_{\mathrm{D}}\right)$ declines toward RA5. This trend solves the above-mentioned contradiction in the dependence on $n$ shown in Figure 3, i.e., (a) and (b) vs. (c). Complementally, the efficiency of triplet generation $\left(\phi_{\mathrm{T}}\right)$ increases toward RA5, which enhances singlet-triplet annihilation to be described in Section 2.1. Finally, we will propose the mechanisms of charge-separation and charge-recombination, which generates the radical-cation and triplet species of RA and CAs on the surface of $\mathrm{TiO}_{2}$ nanoparticles. Figure 7 presents the energies of the singlet, triplet and redox states of RA5 and CA6-CA11, in reference to that of the conduction-band edge (CBE) of $\mathrm{TiO}_{2}$ [4]. Importantly, the energy gap between the $\mathrm{CBE}$ and the $\mathrm{T}_{1}$ levels decreases toward RA5, which explains the increasing order of the triplet generation mentioned above.

Table 3. The time constants of transformation from the $\mathrm{D}_{0}{ }^{\bullet+}-\mathrm{T}_{1}$ complex to the $\mathrm{D}_{0}{ }^{\bullet+}$ and $\mathrm{T}_{1}$ states $\left(k_{\mathrm{d}}^{-1}\right.$ and $\left.k_{\mathrm{t}}^{-1}\right)$ and the $\mathrm{D}_{0}{ }^{\bullet+}$ and $\mathrm{T}_{1}$ lifetimes $\left(k_{\mathrm{d} 0}{ }^{-1}\right.$ and $\left.k_{\mathrm{t} 0}{ }^{-1}\right)$. The partition efficiencies from the $\mathrm{D}_{0}{ }^{\bullet+}-\mathrm{T}_{1}$ complex to the $\mathrm{D}_{0}{ }^{\bullet+}$ and $\mathrm{T}_{1}$ states $\left(\phi_{\mathrm{D}}\right.$ and $\left.\phi_{\mathrm{T}}\right)$ are also listed (reprinted from [7] with permission of MDPI Publishing).

\begin{tabular}{|c|c|c|c|c|}
\hline & $\mathrm{RA5}^{-\mathrm{TiO}_{2}}$ & $\mathrm{CA}-\mathrm{TiO}_{2}$ & $\mathrm{CA} 7-\mathrm{TiO}_{2}$ & $\mathrm{CA}^{-\mathrm{TiO}_{2}}$ \\
\hline$k_{\mathrm{d}}^{-1}(\mu \mathrm{s})$ & 34 & 22 & 9.4 & 5.9 \\
\hline$k_{\mathrm{t}}^{-1}(\mu \mathrm{s})$ & 3.1 & 2.7 & 2.1 & 2.0 \\
\hline$k_{\mathrm{t} 0}^{-1}(\mu s)$ & 22 & 18 & 12 & 9.0 \\
\hline$k_{\mathrm{d} 0}{ }^{-1}(\mu \mathrm{s})$ & $\sim 50$ & $\sim 150$ & $\sim 150$ & $\sim 150$ \\
\hline$\phi_{D}(\%)$ & 8 & 11 & 18 & 25 \\
\hline$\phi_{T}(\%)$ & 92 & 89 & 82 & 75 \\
\hline
\end{tabular}

Figure 8 proposes the excited-state dynamics in a typical CA that is bound to $\mathrm{TiO}_{2}$ [4]: (i) Process $\mathbf{0} \rightarrow \mathbf{1}$ : Upon absorption of photon, electron is transferred to a higher singlet level $\left(\mathrm{S}_{1}\right)$. (ii) Process $\mathbf{1} \rightarrow{ }^{1}$ 2: Electron injection takes place to generate a charge-separated state having a singlet character on the $\mathrm{CA}-\mathrm{TiO}_{2}$ boundary. (iii) ${ }^{1} \mathbf{2} \rightarrow \mathbf{6}$ : the electron is transferred further into $\mathrm{TiO}_{2}$ to form a stable charge-separated state. (iv) $\mathbf{6} \rightarrow \mathbf{0}$ : the reverse electron transfer followed by charge recombination takes place to relax into the ground state. This is a series of changes among the singlet-excited and redox states having a singlet character. 
Figure 7. The energies of the singlet, triplet and redox states of RA5 and CA6-CA11 in comparison to that of the conduction-bond edge $(\mathrm{CBE})$ of $\mathrm{TiO}_{2}$ (reprinted from [4] with permission of the American Chemical Society).

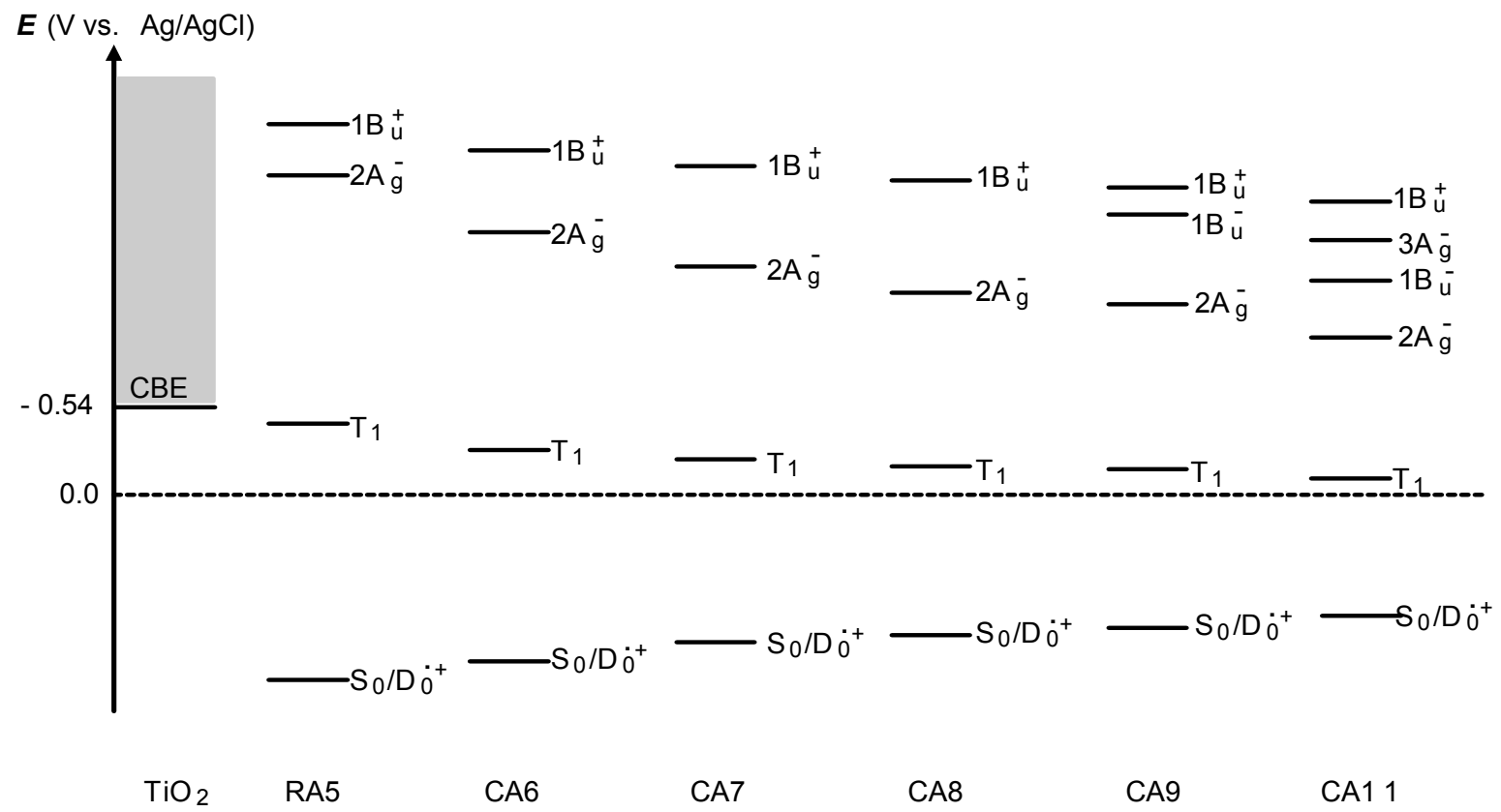

Figure 8. Excitation, electron transfer and relaxation dynamics in a typical RA or CA bound to $\mathrm{TiO}_{2}$ nanoparticles in suspension. Mechanisms of electron injection as well as charge recombination, following intersystem crossing and exciplex formation, to generate triplet $\left(\mathrm{T}_{1}\right)$ and radical cation $\left(\mathrm{D}_{0}{ }^{\bullet+}\right)$ species of the sensitizer. Each numbered state is expressed by a combination of $\mathrm{TiO}_{2}$ and CA in the ground, redox or excited states (reprinted from [4] with permission of the American Chemical Society).
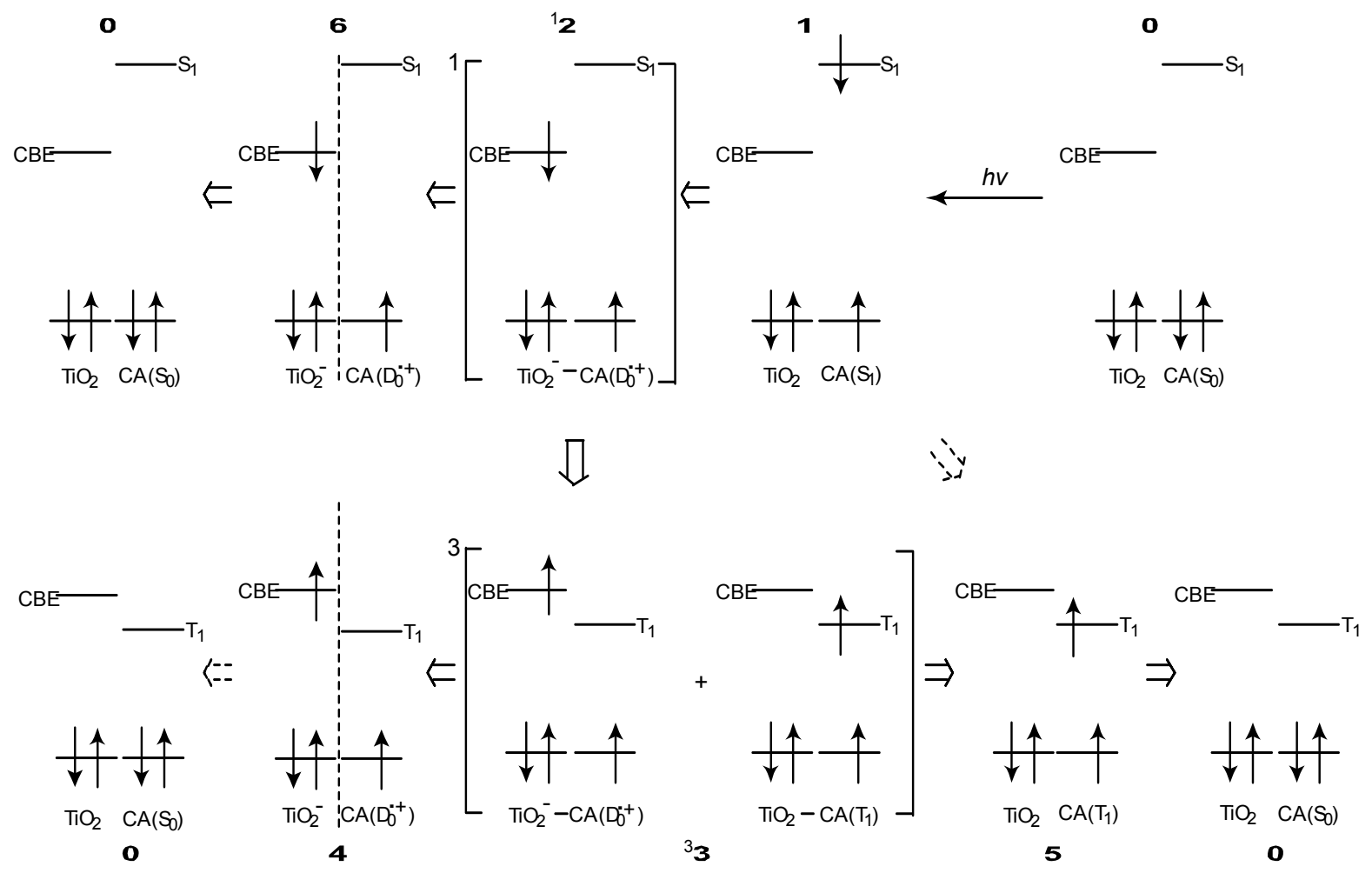
Now, we will consider the generation of the triplet-excited and radical-cation states both having $a$ triplet character in the excited state: (v) Process ${ }^{1} \mathbf{2} \rightarrow{ }^{3} \mathbf{3}$ : When there is a strong spin-orbit coupling in the charge-separated state having the singlet character, it can transform, by the inversion of spin, into the charge-separated state having a triplet character. When the energy gap between the CBE and the $\mathrm{T}_{1}$ levels is small, the resultant charge-separated state can transform further into a charge-transfer complex $\left({ }^{3} 3\right)$ consisting of the charge separated $\left(\mathrm{TiO}_{2}{ }^{-}-\mathrm{CA}\left(\mathrm{D}_{0}{ }^{++}\right)\right)$state and the neutral $\left(\mathrm{TiO}_{2}-\mathrm{CA}\left(\mathrm{T}_{1}\right)\right)$ state. This is exactly what we called 'the combined $\mathrm{D}_{0}{ }^{+}+\mathrm{T}_{1}$ state' (vide supra), because the former component gives rise to the radical-cation electronic absorption, whereas the latter component, the $\mathrm{T}_{1}$-state electronic absorption of CA.

In ${ }^{3} \mathbf{3}$, the relative contribution of the $\mathrm{T}_{1}$-state $\mathrm{CA}$ becomes larger when the energy gap between the $\mathrm{CBE}$ of $\mathrm{TiO}_{2}$ and the $\mathrm{T}_{1}$ states of CA becomes smaller (see Figure 7); this is actually evidenced by the spectral pattern of the $\mathrm{D}_{0}{ }^{-+}+\mathrm{T}_{1}$ state (see Figure 6). This charge-transfer complex can split into two independent components as follows: (vi) ${ }^{3} \mathbf{3} \rightarrow \mathbf{4}$ : It transforms into the pure $\mathrm{D}_{0}{ }^{++}$state of CA, the lifetime of which can be very long when the electron is trapped far from the surface of $\mathrm{TiO}_{2}$ particles in suspension. (vii) ${ }^{3} \mathbf{3} \rightarrow \mathbf{5}$ : it can transform into the $\mathrm{T}_{1}$ state of $\mathrm{CA}$, which decays with an intrinsic $\mathrm{T}_{1}$ lifetime.

Most importantly, the $\mathrm{T}_{1} / \mathrm{D}_{0}{ }^{++}$ratio in the charge-separated state ${ }^{3} \mathbf{3}$ and split states (4 and $\left.\mathbf{5}\right)$ is determined by the gap between the $\mathrm{CBE}$ of $\mathrm{TiO}_{2}$ and the $\mathrm{T}_{1}$ state of RA and CAs. In summary, the mechanisms of electron injection immediately after excitation to the $1 \mathrm{~B}_{\mathrm{u}}^{+}\left(\mathrm{S}_{1}\right)$ state and the charge recombination of the $\mathrm{TiO}_{2}^{-}$- $\mathrm{Car}\left(\mathrm{D}_{0}{ }^{-+}\right)$pair to form triplet Car, after the intersystem crossing and the formation of charge-transfer complex, have been revealed by the analysis of the ps and $\mu$ s timeresolved data obtained by pump-probe spectroscopy of RA and CAs bound to $\mathrm{TiO}_{2}$ nanoparticles in suspension. The conjugation-length $(n)$ dependence of the initial excited-state dynamics has nicely explained the photocurrent and conversion efficiency of solar cells using the RA and CA sensitizers, i.e., the maximum at $n=5$ and the decline toward $n=11$. On the other hand, the decline toward $n=5$ has been explained in terms of the generation of radical cation at later stages. Another possibility of 'singlet-triplet annihilation' using the $\mathrm{T}_{1}$ state generated will be examined in the following Section 2.1.

\section{Singlet-Triplet Annihilation Mechanism; Quenching Singlet Excitation of RA and CAs}

\subsection{Dependence of Photocurrent/Conversion Efficiency on the Dye Concentration in CA7-Sensitized Solar Cells}

Figure 9 shows a set of $I-V$ curves of CA7-sensitized solar cells, when the sensitizer was diluted with a spacer, deoxycholic acid. Figure 10a shows the concentration dependence of $J_{\mathrm{sc}}$ and $\eta$ [3]. Both parameters exhibit similar and unique concentration dependence, which can be characterized as follows: (i) At 100\%, these values are medium among all the values at the different concentrations. (ii) On going from $100 \%$ to $90 \%$, the values exhibit a sudden drop. (iii) Then, they increase up to a maximum at $70 \%$. (iv) From $70 \%$ down to $30 \%$, the values gradually decrease. (v) Below $30 \%$, they decrease steeply toward the values at $10 \%$. 
Figure 9. Concentration dependence of the $I-V$ curves in CA7-sensitized solar cell (reprinted from [3] with permission of Elsevier).

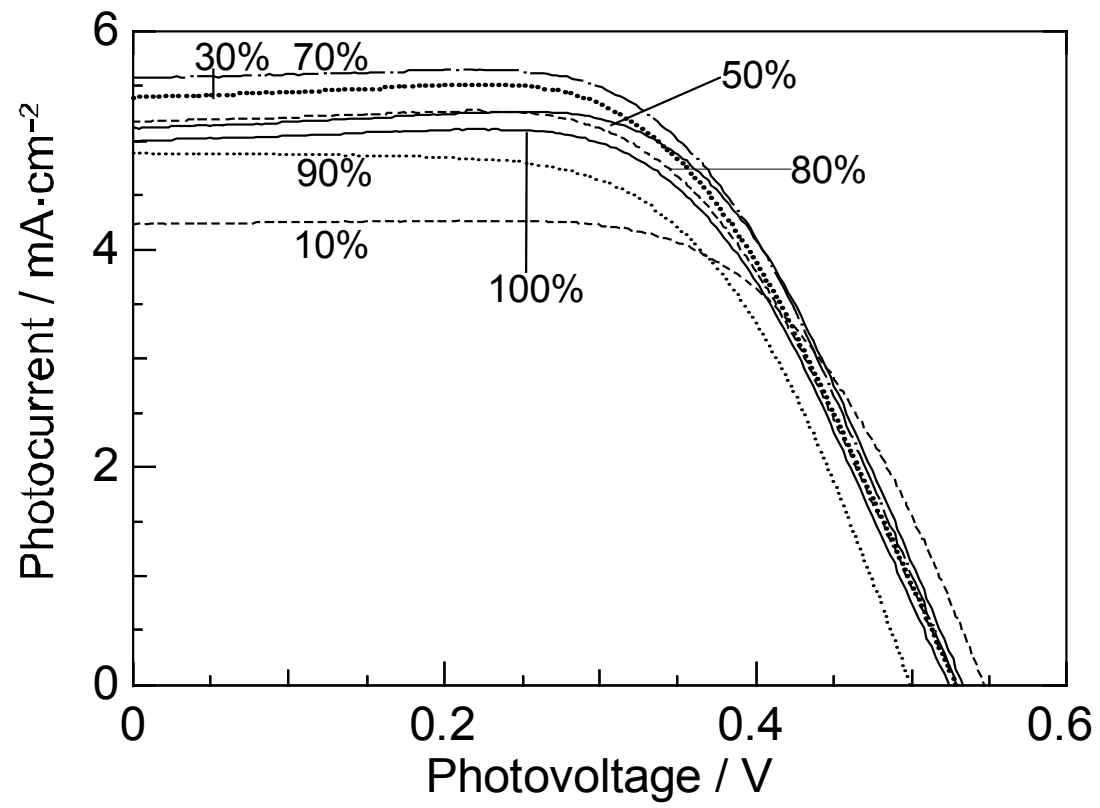

Figure 10. Effects of dilution with a spacer (deoxycholic acid) on (a) the photocurrent $\left(J_{\mathrm{sc}}\right)$ and conversion efficiency $(\eta)$ and (b) the relative photocurrent $\left({ }^{\mathrm{r}} J_{\mathrm{sc}}\right)$ and conversion efficiency $\left({ }^{\mathrm{r}} \eta\right)$ of CA7-sensitized solar cells. To obtain ${ }^{\mathrm{r}} J_{\mathrm{sc}}(\mathrm{X})$ at a mole fraction $\mathrm{X}$, for example, $J_{\text {sc }}(\mathrm{X})$ was scaled against concentration, and, then, a ratio was taken in reference to the value with no dilution. Thus, ${ }^{\mathrm{r}} J_{\mathrm{sc}}(\mathrm{X})=J_{\mathrm{sc}}(\mathrm{X}) / \mathrm{X} / J_{\mathrm{sc}}(\mathrm{X}=1)$ and ${ }^{\mathrm{r}} \eta(\mathrm{X})=\eta(\mathrm{X}) / \mathrm{X} / \eta(\mathrm{X}=1)$ (reprinted from [3] with permission of Elsevier).

(a)
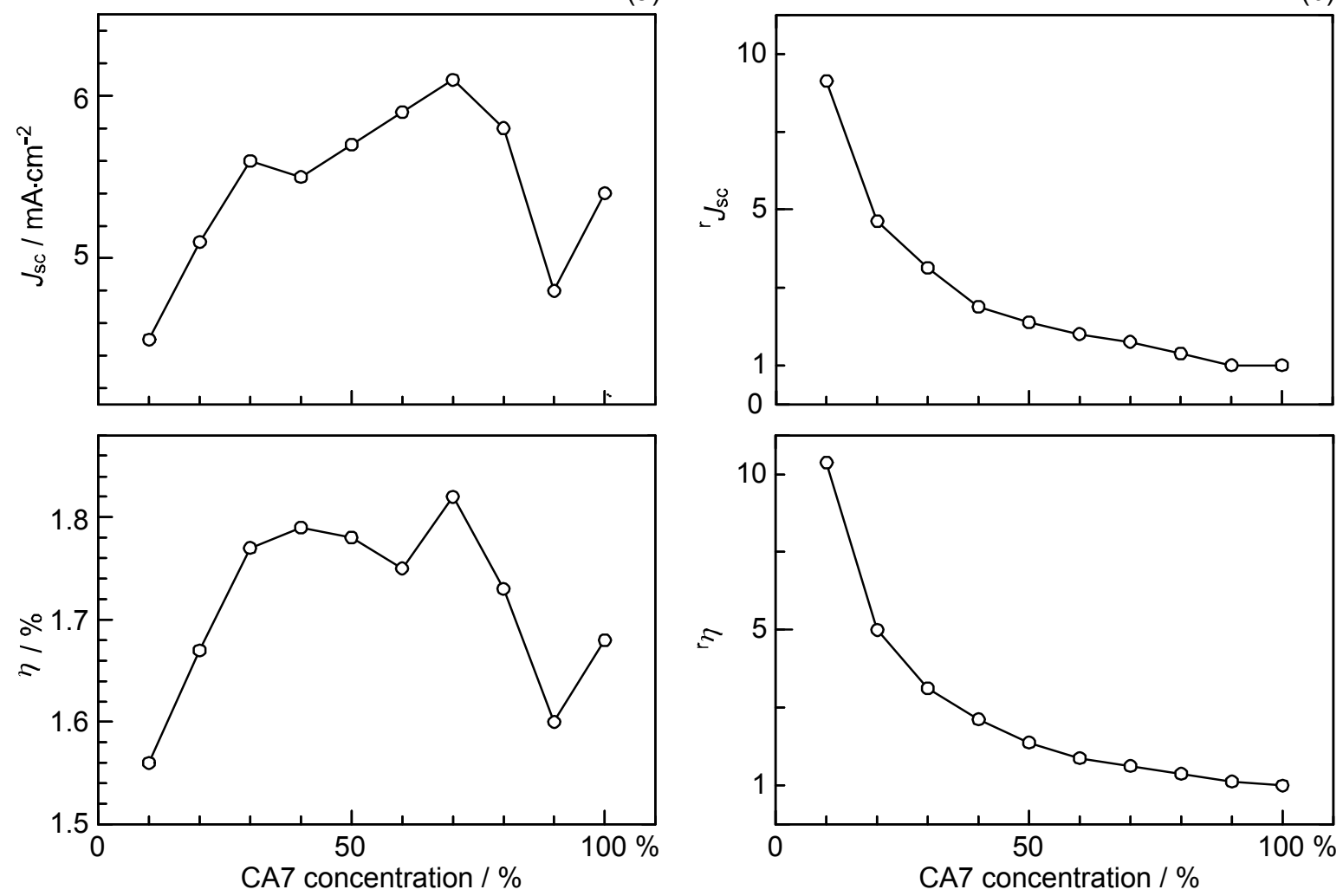
We propose four different forms of excitation based on the results shown in Figure 11 [3], where the dye molecules (०) are diluted with the spacer molecules (•): (i) at 100\%, a coherent excitonic excitation takes place in an aggregate of dye molecules (we call this 'coherent delocalized excitation'). (ii) At $90 \%$, this excitation is destroyed by a spacer molecule that functions as a defect. (iii) At $70 \%$, a localized excitation on a single molecule can migrate from one to another in different directions. This 'migrating excitation' must become most efficient when the dye concentration becomes around $2 / 3$, because branched routes for the migrating excitation are formed. (iv) At 30\%, the dye molecules become isolated being intervened by a larger number of spacer molecules. This 'isolated excitation' must become the largest in number when the dye concentration becomes around $1 / 3$.

Figure 11. Typical arrangements of the dye (०) and spacer $(\bullet)$ molecules on the $\mathrm{TiO}_{2}$ surface formed during the processes of dilution of the former with the latter (reprinted from [3] with permission of Elsevier).

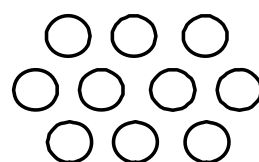

$100 \%$

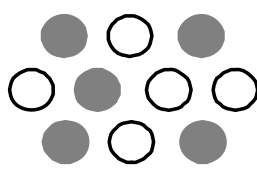

$50 \%$

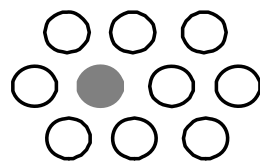

$90 \%$

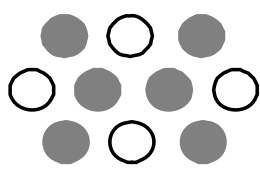

$40 \%$

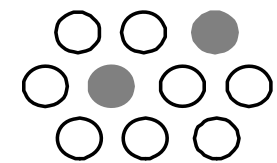

$80 \%$

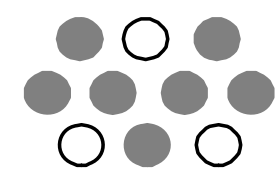

$30 \%$

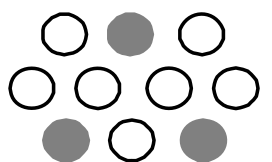

$70 \%$

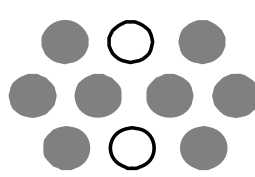

$20 \%$

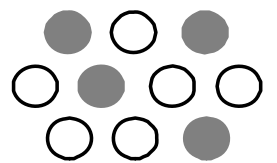

$60 \%$

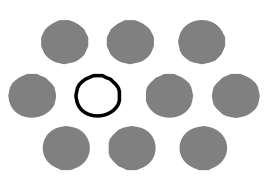

$10 \%$

Based on the above three different types of singlet excitation on the $\mathrm{TiO}_{2}$ layer and the generation of the triplet state, and the resultant singlet-triplet annihilation as an intrinsic property of CAs bound to $\mathrm{TiO}_{2}$, we propose a possible mechanism to explain the unique concentration dependence of photocurrent/conversion efficiency in the fabricated CA7-sensitized solar cell (see Figure 10a and Figure 11): (i) In a coherent delocalized excitation at $100 \%$, there is a good chance that the widelyexpanded singlet excitation reaches a dye molecule in the $\mathrm{T}_{1}$ state to cause singlet-triplet annihilation. (ii) In a partially-destroyed delocalized excitation at $90 \%$, the advantage of the widely-expanded coherent excitation in electron injection is lost, but there is still a chance of collision between 'a partially-expanded delocalized singlet excitation' and a localized triplet excitation to annihilate the former. (iii) In the localized excitation migrating along one of the branched routes at $\sim 70 \%$, there is a much less chance of collision with a triplet excitation, unless it is located on the particular route. (iv) In an isolated singlet excitation, there is no chance of its collision with an isolated triplet excitation. Then, the photocurrent/conversion efficiency decreases linearly with the decreasing number of the localized excited-dye molecules.

The relative photocurrent $\left({ }^{\mathrm{r}} J_{\mathrm{sc}}\right)$ and conversion efficiency $\left({ }^{\mathrm{r}} \eta\right)$ are depicted in Figure $10 \mathrm{~b}$ (see the caption for their definition). Their concentration dependence indicates that changes in the singlet excitation of the dye molecules take place continuously, and the relative performance $\left({ }^{\mathrm{r}} J_{\mathrm{sc}}\right.$ and $\left.{ }^{\mathrm{r}} \eta\right)$ becomes systematically enhanced until 9-10 times on going from the first to the last form of singlet excitation. 
To summarize, the dependence of the photocurrent and conversion efficiency of the CA7-sensitizerd solar cell, on the dye concentration, has been explained in terms of changes in the form of singlet excitation of the sensitizer molecules on the surface of $\mathrm{TiO}_{2}$ layer, i.e., coherent delocalized excitation $\rightarrow$ localized migrating excitation $\rightarrow$ isolated excitation. There is a good chance of substantial enhancement in performance, if we succeeded in achieving only the localized excitation, keeping the total number of excited-state dye molecules the same.

The substantially reduced performance at the $100 \%$ dye concentration is ascribable to singlet-triplet annihilation reaction. Therefore, the decrease in the photocurrent/conversion efficiency of solar cells from the CA7 sensitizer toward the RA5 sensitizer (see Figures 3a,b) can now be explained also by the effect of singlet-triplet annihilation among the sensitizer molecules on the surface of the $\mathrm{TiO}_{2}$ layer, in addition to the effect of the increasing triplet generation described in Section 1.

\subsection{Suppression of Photocurrent/Conversion Efficiency in Polyene Sensitizers Having Higher} Polarizability (the Higher Trend of Aggregate Formation)

We prepared a set of four sensitizers having different polarizabilities and, as a result, different tendency of aggregated formation, and examined changes in the photocurrent/conversion efficiency of fabricated solar cells, depending on the dye concentration and the light intensity. The most-aggregateforming dye exhibited the enhancement of performance by lowering the dye concentration and the light intensity, supporting the idea of singlet-triplet annihilation:

Figure 12 shows the structures of four different polyene sensitizers that were used for fabricating the solar cells [8]. The common skeleton of the sensitizers is the benzene ring connected to a short polyene $(n=6)$, to the end of which an electron-withdrawing carboxyl group is attached ( $\phi-6-\mathrm{CA})$; to the opposite end of the benzene ring the MeO-, $(\mathrm{MeO})_{3}-$ or $\mathrm{Me}_{2} \mathrm{~N}$ - electron-donating group is attached to realize systematically the electron push-pull system in the latter set of sensitizers.

The set of polyene sensitizers are named $\phi-6-\mathrm{CA}, \mathrm{MeO}-\phi-6-\mathrm{CA},(\mathrm{MeO})_{3}-\phi-6-\mathrm{CA}$ and $\mathrm{Me}_{2} \mathrm{~N}-\phi-6-$ $\mathrm{CA}$; the polarizability of polyene to enhance van der Waals intermolecular interaction to form aggregates is supposed to increase in this order. Actually, the transition-dipole moment calculated by the use of the molar extinction coefficient $(\varepsilon)$ was in the order, 14.2, 15.1, 15.2 and 15.6 D, and the tendency of aggregate formation, judged by the blue-shift of the $1 \mathrm{~B}_{\mathrm{u}}{ }^{+}$absorption band, was seen in the same order (data not shown).

Figure 13a shows the concentration dependence of the $I-V$ curves of solar cells using the above set of sensitizers. In the least-polarizable sensitizer, $\phi$-6-CA, the photocurrent $\left(J_{\mathrm{sc}}\right)$ was the highest at $100 \%$ and monotonously decreased toward the lower concentration.

In the most-polarizable sensitizer, $\mathrm{Me}_{2} \mathrm{~N}-\phi-6-\mathrm{CA}$, on the other hand, the photocurrent was the lowest at $100 \%$ and monotonously increased toward the lower concentration. The latter change is contrary to our expectation, and can be explained only in terms of singlet-triplet annihilation. At 100\%, the delocalized excitonic excitation should be generated due to the aggregate formation, which can be readily annihilated by collision with the triplet species within the expanded, excitonically-excited region. The chance of this singlet-triplet annihilation must become smaller by lowering the dye concentration. 
Figure 12. Chemical structures of a set of four polyene sensitizers with the increasing polarizability and, as a result, the increasing tendency of aggregate formation (reprinted from [8] with permission of Elsevier).

transition dipole momen<smiles>CC(/C=C/C=C/C(C)=C/C=C/c1ccccc1)=C\C=C\C(=O)O</smiles><smiles>COC(=O)OC</smiles><smiles>COC(=O)/C(C)=C/C=C/C=C(C)/C=C/C=C(C)/C=C/c1c(OC)cc(OC)cc1OC</smiles><smiles>CC(/C=C/C=C(C)/C=C/c1ccc(N(C)C)cc1)=C\C=C\C=C(/C)C(=O)O</smiles>

Figure 13. (a) The concentration dependence and (b) the light-intensity dependence (at two different concentrations) of the $I-V$ curves in solar cells using the four sensitizers having different polarizabilities (see Figure 12) (reprinted from [8] with permission of Elsevier).
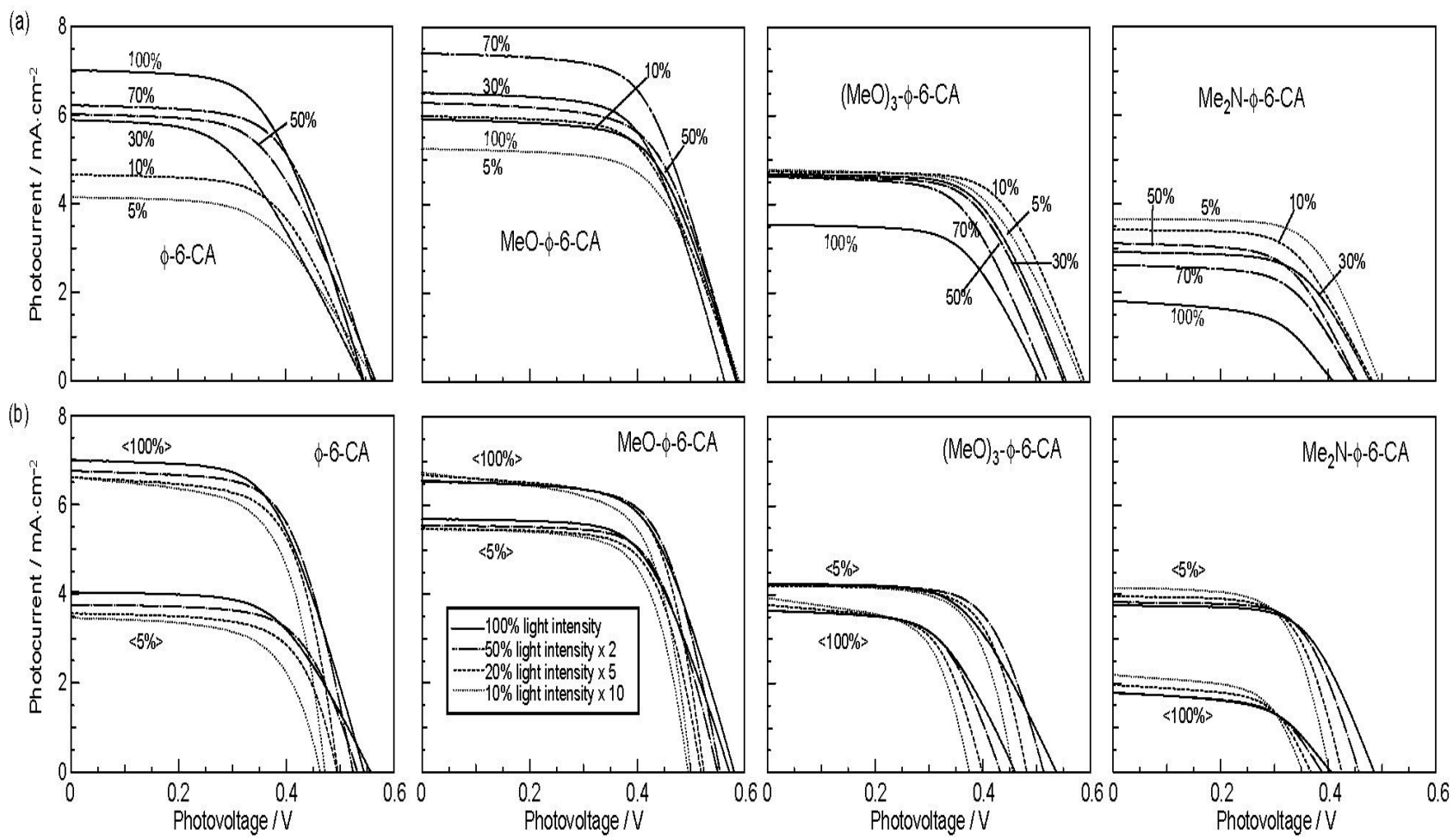
Figure $13 \mathrm{~b}$ shows the dependence of the $I-V$ curves of the solar cells on the light intensity at two different dye concentrations (5\% and 100\%) [8]. In the least-polarizable sensitizer, $\phi-6-\mathrm{CA}$, the photocurrent decreased with the lowering light intensity. On the other hand, in the most-polarizable sensitizer, $\mathrm{Me}_{2} \mathrm{~N}-\phi-6-\mathrm{CA}$, the photocurrent increased, instead, with the lowering light intensity. The latter change is contrary to our expectation, and can be explained only in terms of singlet-triplet annihilation, because the generation of both the singlet and triplet excitation must become suppressed at the lower light intensity.

Figure 14a plots the concentration dependence of conversion efficiency $(\eta)$ for the set of polyene sensitizers [8]. In the least-polarizable sensitizer, $\phi-6-\mathrm{CA}$, the conversion efficiency monotonously decreased, while in the most-polarizable sensitizer, $\mathrm{Me}_{2} \mathrm{~N}-\phi-6-\mathrm{CA}$, it monotonously increased with the lowering dye concentration. In the second-least polarizable sensitizer, $\mathrm{MeO}-\phi-6-\mathrm{CA}$, conversion efficiency exhibits a maximum at $70 \%$, while in the second-most polarizable sensitizer, $(\mathrm{MeO})_{3}-\phi-6-\mathrm{CA}$, it exhibits a maximum at 5\%. Figure $14 \mathrm{~b}$ shows that ${ }^{\mathrm{r}} \eta$ increased in the order, $\phi$-6-CA $<\mathrm{MeO}-\phi-6-\mathrm{CA}<(\mathrm{MeO})_{3}-\phi-6-\mathrm{CA}<\mathrm{Me}_{2} \mathrm{~N}-\phi-6-\mathrm{CA}$; it is $\sim 60$ times in the last sensitizer [7].

Figure 14. Concentration dependence of (a) the conversion efficiency $(\eta)$ and (b) the relative conversion efficiency $\left({ }^{\mathrm{r}} \eta\right)$ in solar cells using the four sensitizers with increasing polarizabilities (reprinted from [8] with permission of Elsevier).
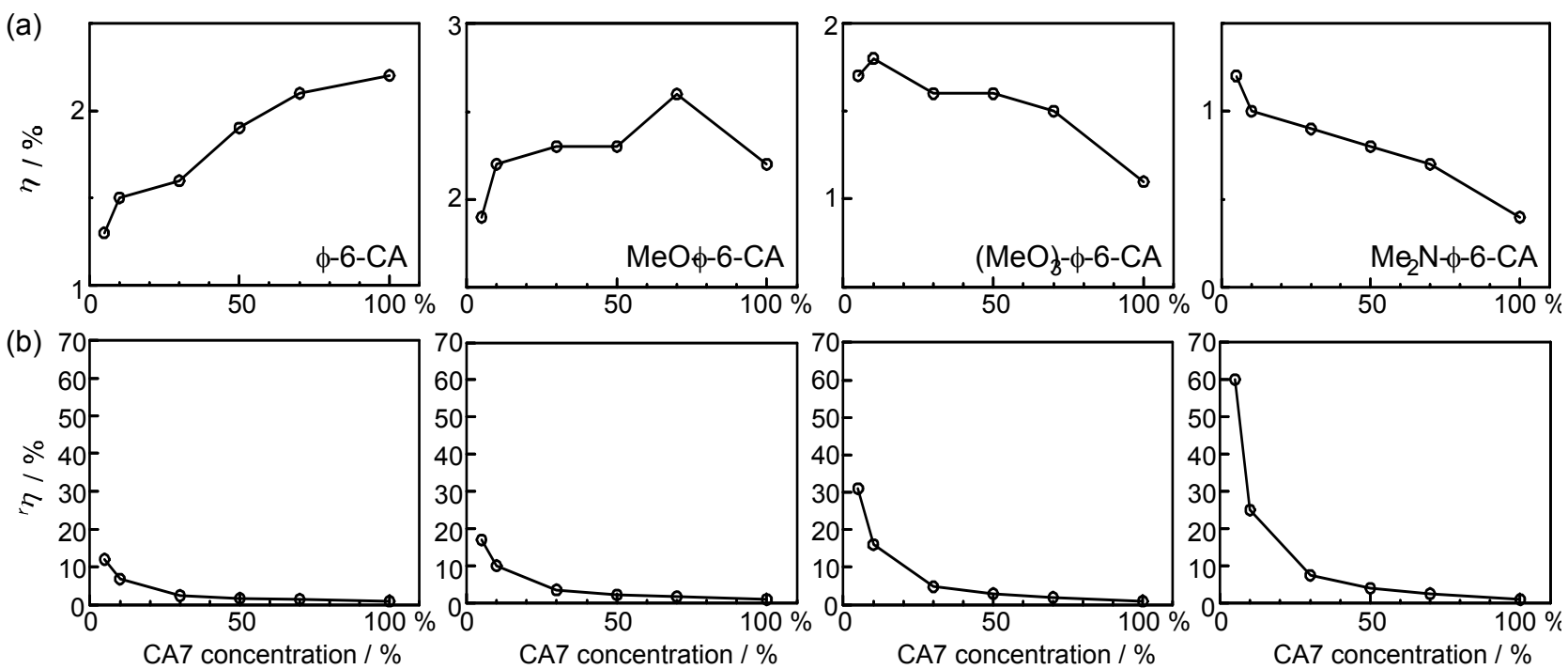

To summarize, the absence or presence of singlet-triplet annihilation has been demonstrated by lowering the dye concentration and the light intensity in solar cells by the use of the four sensitizers having the increasing polarizability and, as a result, the increasing tendency of aggregate formation. The least polarizable (the least aggregate-forming) sensitizer gave rise to the decreasing conversion efficiency with the decreasing dye concentration and light intensity, whereas the most polarizable (the most aggregate-forming) sensitizer gave rise to the increasing conversion efficiency with the decreasing dye concentration and light intensity. The four different patterns, in the dependence on the dye concentration and the light intensity, can be used as a standard to examine the degree of aggregate formation and the absence or presence of singlet-triplet annihilation in a new sensitizer. 


\section{Enhancement of Photocurrent/Conversion Efficiency by Preventing Singlet-Triplet Annihilation in Car-Phe Adduct and $\mathrm{Chl} c_{2}$ Sensitizers}

\subsection{Pheophorbide-Car Adduct: Energy Transfer and Electron Transfer from the Car to Phe Moiety}

While searching for a sensitizer of Chl $a$ derivative having a cyclic conjugated system, we found that pheophorbide $a$ (Phe $a$ ) having the chlorin skeleton gave rise to reasonably-high photocurrent and conversion efficiency. Electron transfer from a neutral Car to Phe $a$ radical cation (Phe $a^{\bullet+}$ ) can prevent the charge recombination in the $\mathrm{TiO}_{2}^{-}{ }^{-} \mathrm{Car}^{\bullet+}$ charge-separated state, in the order, $\mathrm{TiO}_{2}{ }^{-} \cdots \mathrm{Phe}^{+}-\mathrm{Car} \rightarrow \mathrm{TiO}_{2}^{-} \cdots$ Phe-Car ${ }^{\bullet+}$. Actually, the Car spacers enhanced the photocurrent and conversion efficiency of a solar cell using a Phe sensitizer.

We found no signs of singlet-energy transfer in the above experiments, even by the use of the shortest-chain Cars ( $n=8$ and 9$)$ having the higher singlet energies $\left(1 \mathrm{~B}_{\mathrm{u}}{ }^{+}\right)$than those of Phe $a\left(\mathrm{Q}_{x}\right)$ (see Figure 15 [7]).

Figure 15. The energies of the optically-allowed $1 \mathrm{~B}_{\mathrm{u}}^{+}$and the optically-forbidden $2 \mathrm{~A}_{\mathrm{g}}{ }^{-}$, $1 \mathrm{~B}_{\mathrm{u}}{ }^{-}$and $3 \mathrm{~A}_{\mathrm{g}}{ }^{-}$states of Cars and those of the $\mathrm{Q}_{\mathrm{x}}$ and $\mathrm{Q}_{\mathrm{y}}$ states of Phe $a$ (Phe $y$ ). Shorterchain Cars ( $n=8$ and 9) have a better chance of singlet-energy transfer from Car to Phe $a$ $\left(1 \mathrm{~B}_{\mathrm{u}}^{+} \rightarrow \mathrm{Q}_{x}\right)$ (reprinted from [7] with permission of MDPI Publishing).

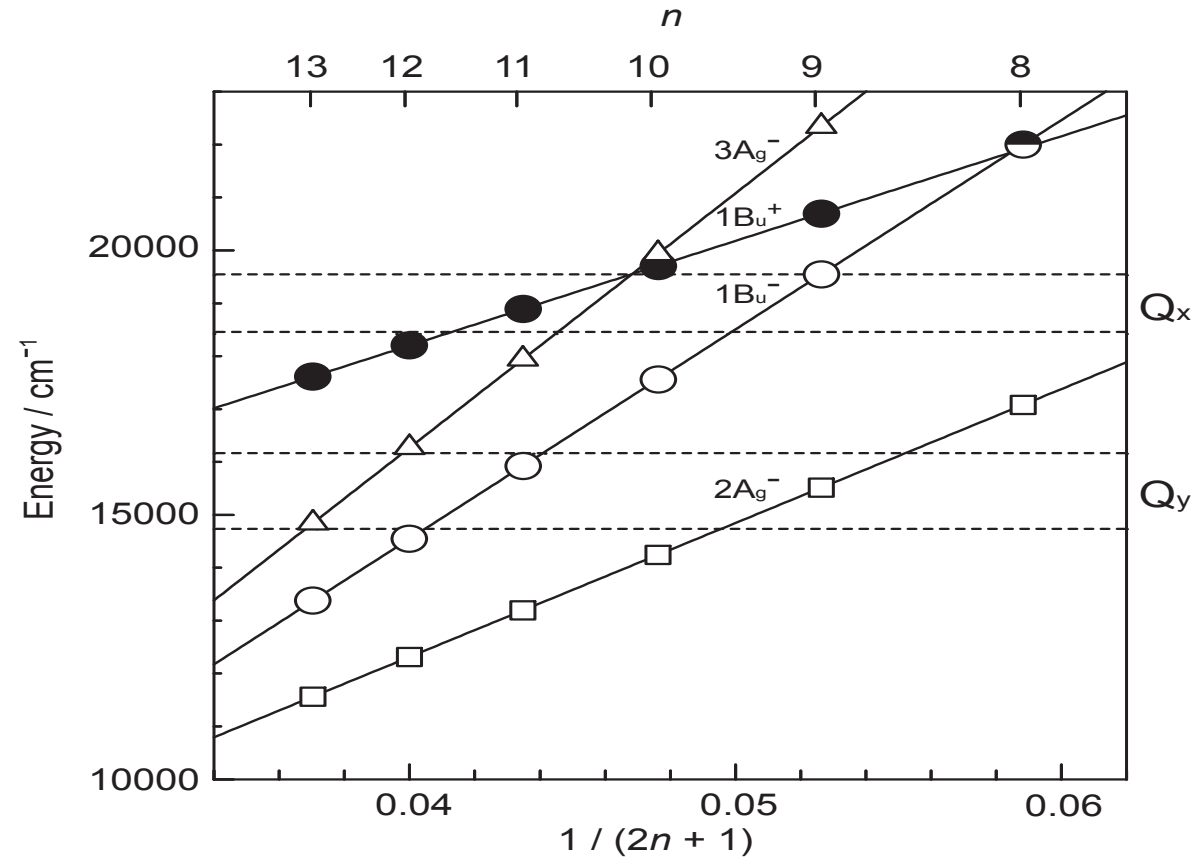

We suspected that the direct van der Waals contact and the correct orientations of the transition dipoles between the Car and the Phe $a$ moieties may be necessary to facilitate efficient singlet-energy transfer. Then, we synthesized an adduct sensitizer consisting of Phe $y$ (modified from Phe $a$ ) and Car, which actually realized the singlet-energy transfer from the Car to the Phe moiety (see Figure 16), in addition to electron transfer, enhancing photocurrent/conversion efficiency. Further, the Car moiety, connected by single bonds to the Phe $y$ moiety, could prevent the aggregate formation and the resultant singlet-triplet annihilation, which was evidenced by the suppression of performance by lowering the light intensity. 
Figure 16. Chemical structures of Phe $y$ and Phe-Car adduct (reprinted from [9] with permission of Elsevier).

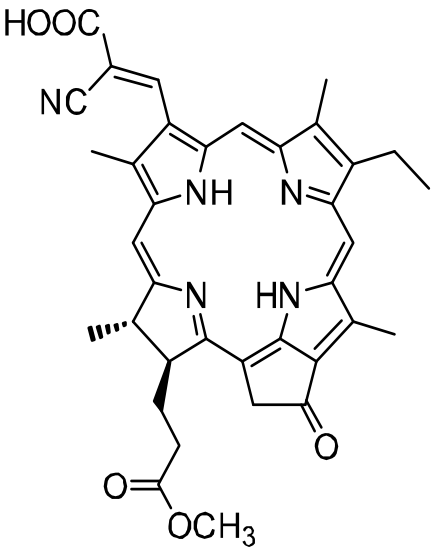

Phe $y$

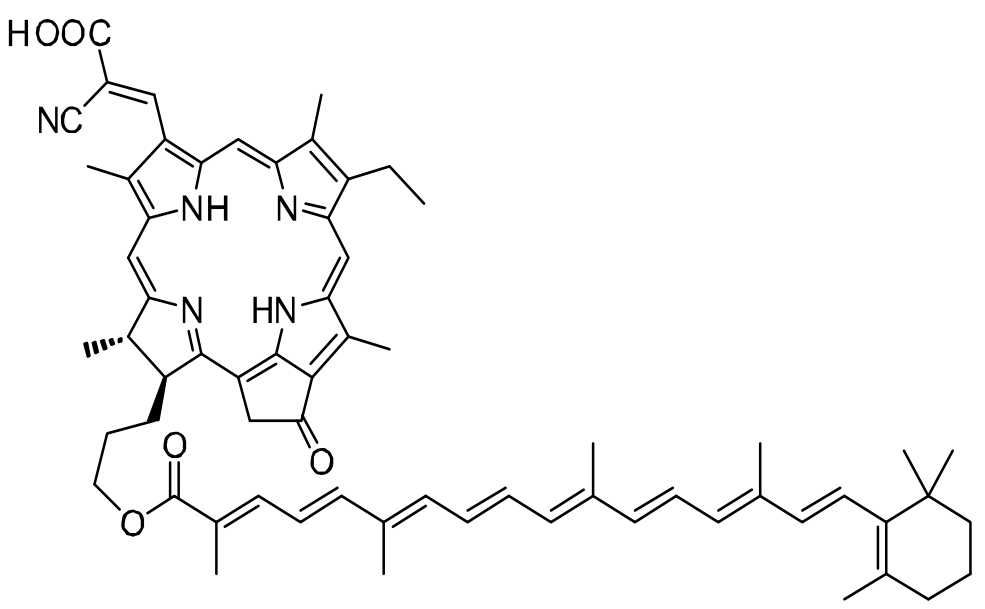

Phe-Car adduct

Figure 16 presents the chemical structures of 'Phe $y$ ' sensitizer and 'Phe-Car adduct' [9]. Phe $y$ has a structure similar to Phe $a$, in which the carboxyl group attached to ring $\mathrm{A}$ is replaced by the ethenylcyano-carboxyl group that was supposed to enhance electron injection. Phe-Car adduct consists of the Phe $y$ and $\beta$-apo-8'-carotenoyl $(n=9)$ moieties, and, therefore, singlet energy-transfer becomes possible from the latter to the former.

In fact, the $\pi$-conjugated systems of the two moieties are connected loosely through a couple of single bonds so that their electron clouds can overlap with each other to facilitate efficient electron transfer and, in addition, the $1 \mathrm{~B}_{\mathrm{u}}{ }^{+}$transition moment of the Car moiety and the $\mathrm{Q}_{x}$ transition moment of the Phe moiety can be set parallel to facilitate the $1 \mathrm{~B}_{\mathrm{u}}{ }^{+}$to $\mathrm{Q}_{x}$ singlet-energy transfer. (Note that the $\mathrm{Q}_{x}$ transition moment of Phe and the $1 \mathrm{~B}_{\mathrm{u}}^{+}$transition moment of Car should be actually overlapped with each other; the figure is just for simplification.) When the adduct is bound to the $\mathrm{TiO}_{2}$ surface, the intervening bulky Car group may prevent the formation of Phe $y$ aggregate and, as a result, suppress the singlet-triplet annihilation reaction.

Figure 17a compares the IPCE profiles of the solar cells using the Phe $y$ and Phe-Car adduct sensitizers [9]. In the longer-wavelength region (500-800 nm), we see the shift of basically the same IPCE profile from the former to the latter. The shift of the IPCE profile in this region is ascribable to electron transfer from the Car to the Phe $y$ moiety. In the shorter-wavelength region (370-470 nm), a bump is observed in the IPCE profile of Phe-Car adduct. Definitely, this is ascribable to singlet-energy transfer from the Car to the Phe $a$ moiety. Figure $17 \mathrm{~b}$ compares the $I-V$ curves for the two sensitizers [9]: the Phe $y$ sensitizer gives rise to a higher $V_{\text {oc }}$ value, while the adduct sensitizer, a higher $J_{\text {sc }}$ value. The former observation presumably reflects the better packing of the Phe $y$ sensitizers on the $\mathrm{TiO}_{2}$ surface, because the bulky Car moiety in Phe-Car adduct must prevent ordered surface coverage. The latter observation must reflect the larger photocurrent due to the electron transfer and energy transfer from the Car to the Phe moiety as mentioned above. The introduction of the Car moiety enhances $J_{\mathrm{sc}}$ by 1.6 times and $\eta$ by 1.3 times. The $E_{\mathrm{ox}}$ values of Phe-Car adduct reflected those of the Car moiety $(0.95 \mathrm{~V})$ and the Phe $y$ moiety $(1.17 \mathrm{~V})$, which supports the idea of electron transfer from both the Car and the Phe moiety. 
Figure 17. (a) The IPCE profiles and (b) the $I-V$ curves of solar cells sensitized by Phe $y$ and Phe-Car adduct (reprinted from [9] with permission of Elsevier).

(a)

(b)
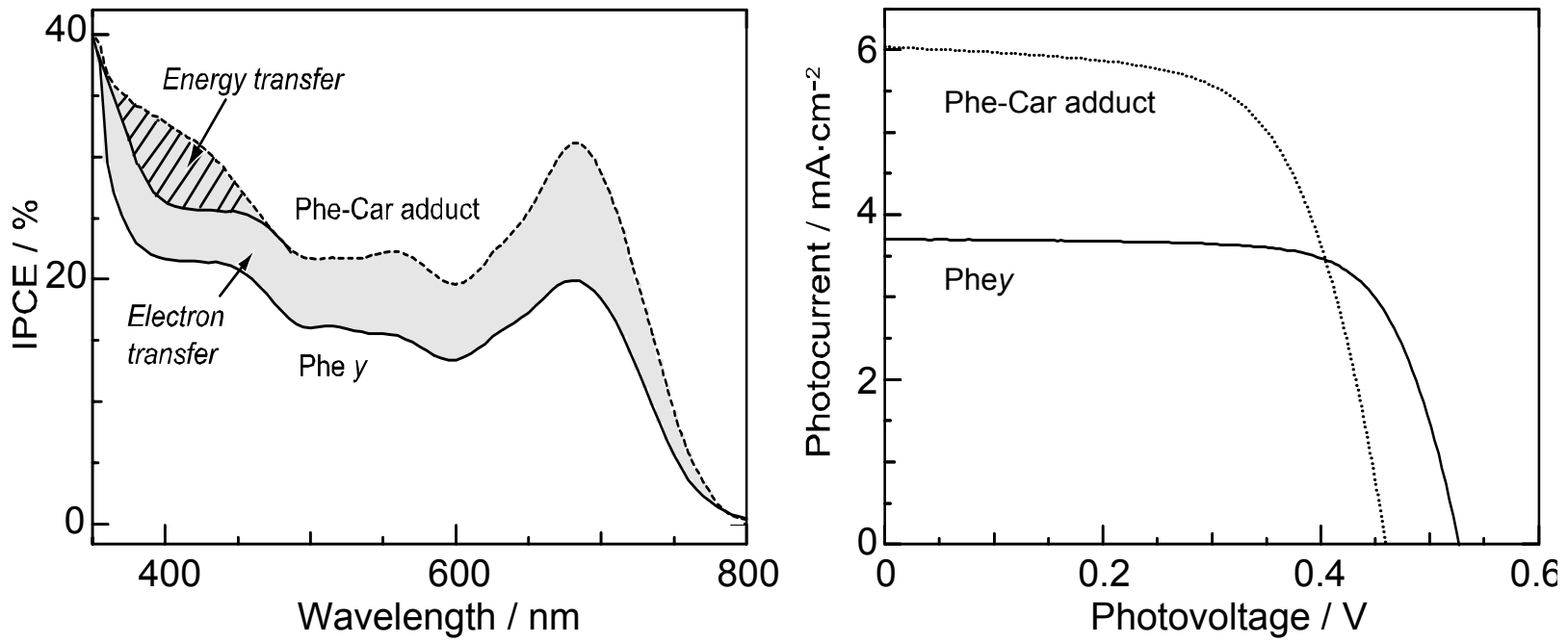

Figure 18 compares the light-intensity dependence of the $I-V$ curves of solar cells using the Phe $y$ and Phe-Car adduct sensitizers [9].

Figure 18. The light-intensity dependence of the $I-V$ curves in solar cells using the Phe $y$ and Phe-Car adduct sensitizers (reprinted from [9] with permission of Elsevier).

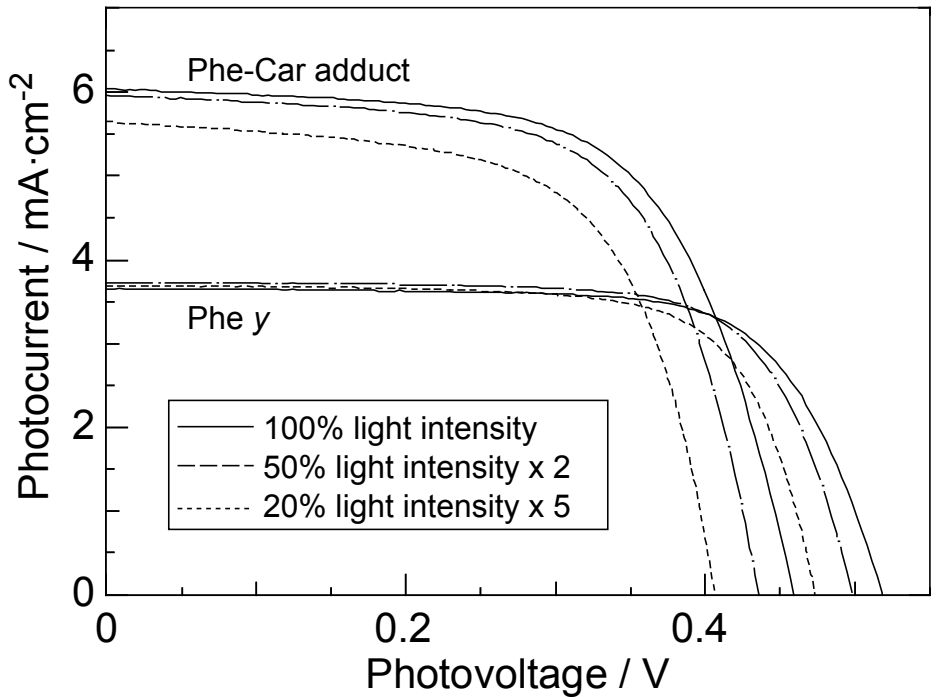

In the former, no clear changes in $J_{\text {sc }}$ is seen even by lowering the light intensity into $1 / 5$, whereas in the latter, systematic decrease in $J_{\mathrm{sc}}$ is seen as expected. The changes are somewhat comparable to the case of polyenes (see Figure 13): the light-intensity dependence of Phe $y$ is similar to that of $(\mathrm{MeO})_{3}-\phi-6-\mathrm{CA}$ (except for $100 \%$ ), whereas that of Phe-Car adduct, to that of $\phi-6$-CA. The results indicate that some aggregation to cause singlet-triplet annihilation is formed in the Phe $y$ sensitizer, whereas practically no aggregates are formed in the Phe-Car adduct sensitizer. Figure 19 pictorially proposes the mechanisms of enhancement in photocurrent/conversion efficiency on going from the Phe $y$ to the Phe-Car adduct sensitizer, which include (i) electron transfer and (ii) singlet-energy transfer 
from the Car to the Phe $y$ moiety as well as (iii) the suppression of the singlet-triplet annihilation reaction by preventing the aggregate formation by the use of the bulky Car moiety as a spacer.

Figure 19. Mechanisms of the enhancement of photocurrent and conversion efficiency in the solar cell using the Phe-Car adduct sensitizer (reprinted from [9] with permission of Elsevier).
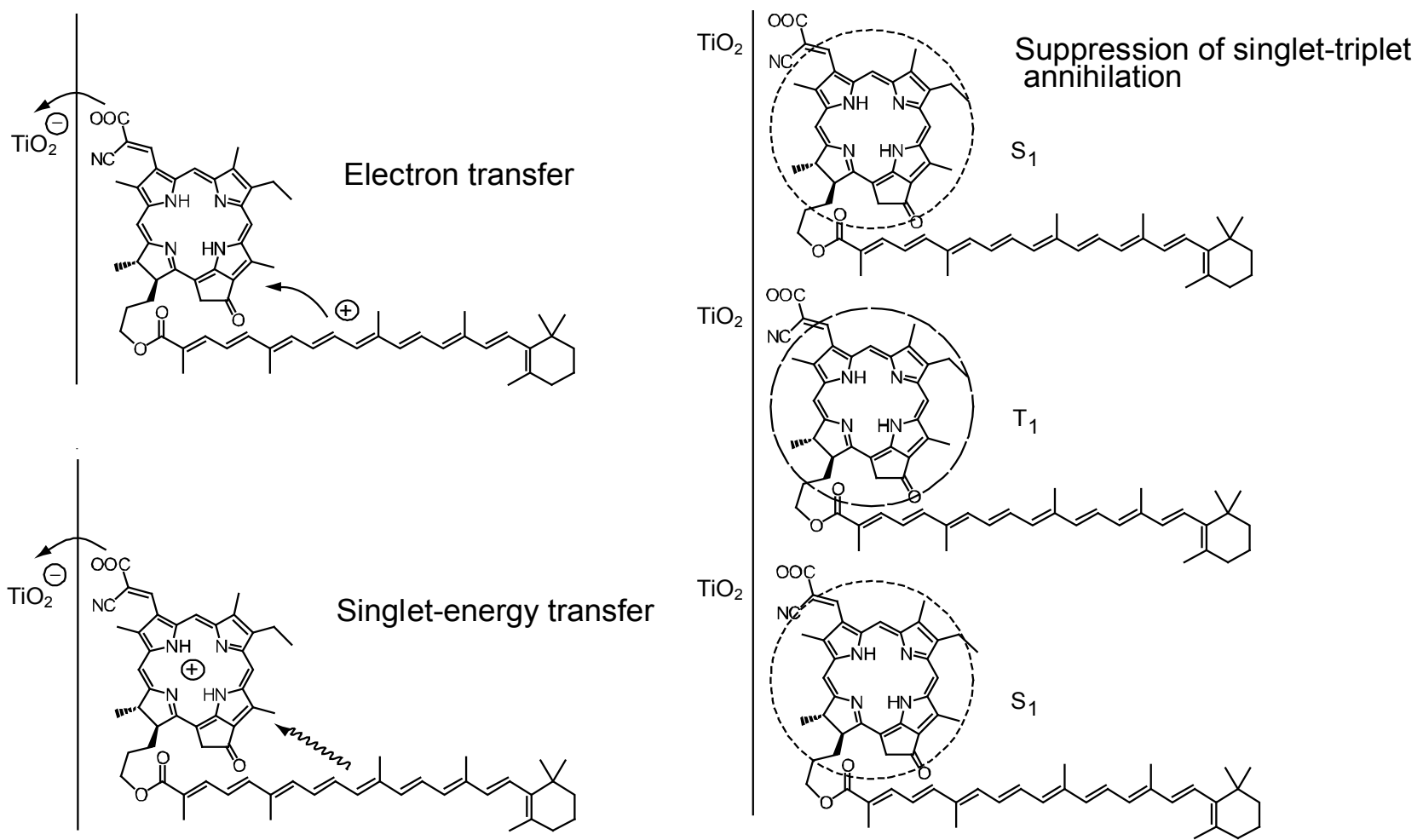

Both singlet-energy transfer and electron transfer from the Car to the Phe moiety have been realized in the Phe-Car adduct. The photocurrent $\left(J_{\text {sc }}\right)$ was enhanced by 1.6 times, the photovoltage $\left(V_{\text {oc }}\right)$ was lowered by 0.9 times and, as a result, the conversion efficiency $(\eta)$ was enhanced by 1.3 times. The $\pi$-conjugated chain of the Car moiety prevented the aggregate formation of the Phe moiety so that no sign of singlet-triplet annihilation was observed. Therefore, the Phe-Car adduct is potentially an excellent sensitizer to be used in a more refined way; the addition of short polyene spacers would improve the coverage of the $\mathrm{TiO}_{2}$ layer and to enhance the photovoltage $\left(V_{\text {oc }}\right)$, for example.

\subsection{Chl c (Mg-Pheophorbide c) Sensitizers Having Porphyrin Skeleton}

Figure 20 presents the chemical structures of the pairs of Chls $c$ and Chls $c$ ' extracted from a sea weed called 'Undaria pinnatifida (Wakame)' [7]. The structures were determined by mass spectrometry and ${ }^{1} \mathrm{H}-\mathrm{NMR}$ spectroscopy. The latter includes rotating-frame Overhauser effect spectroscopy (ROESY) measurement to determine the nuclear Overhauser effect (NOE) correlations [10]. $\mathrm{Chl} c_{1}\left(\mathrm{Chl} c_{1}^{\prime}\right)$ and $\mathrm{Chl} c_{2}\left(\mathrm{Chl} c_{2}^{\prime}\right)$ have an ethyl group and a vinyl group, respectively, attached to ring $\mathrm{B}$ in different conformations. Further, $\mathrm{Chl} c_{1}$ and $\mathrm{Chl} c_{2}$ ( $\mathrm{Chl} c_{1}$ ' and $\mathrm{Chl} c_{2}$ ') have hydrogen (hydroxyl group) attached to ring $\mathrm{E}$, and also the carboxyl group attached to ring $\mathrm{D}$ through the vinyl group in the cis (trans) conformation with respect to a single bond attached to ring D. Thus, Chl $c_{1}$ ' and $\mathrm{Chl} c_{2}$ ' can form intramolecular hydrogen bonding between the hydroxyl and carboxyl groups. 
Importantly, the chemical-shift values of the vinyl $\mathrm{H}$ suggest that the electron density is in the order, $\mathrm{Chl} c_{2}>\operatorname{Chl} c_{1}>\mathrm{Chl} c_{2}{ }^{\prime}>\mathrm{Chl} c_{1}$ '.

Figure 20. Chemical structures of $\mathrm{Chl} c_{1}, \mathrm{Chl} c_{2}, \mathrm{Chl} c_{1}^{\prime}$ and $\mathrm{Chl} c_{2}$ ' (reprinted from [7] with permission of MDPI Publishing).

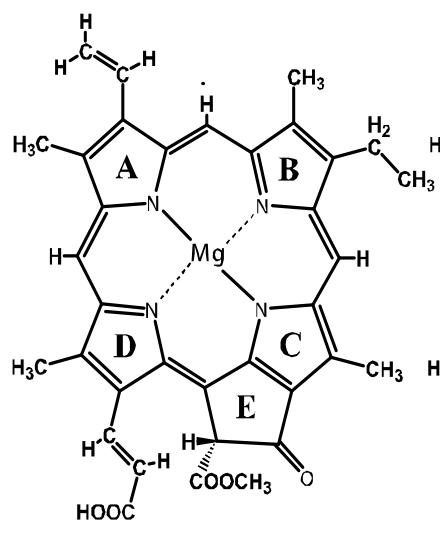

Chl $c_{1}$

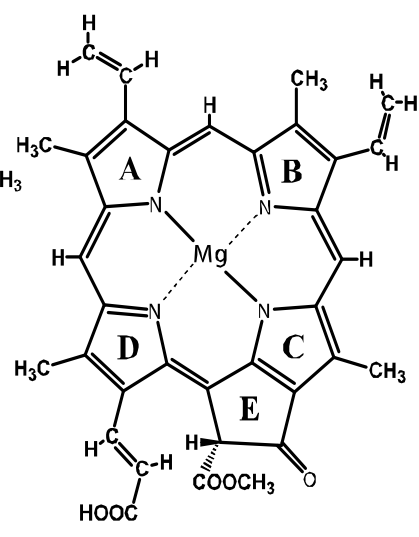

Chl $c_{2}$

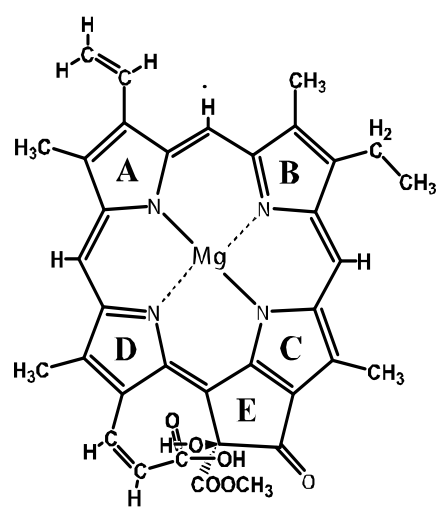

Chl $c 1^{\prime}$

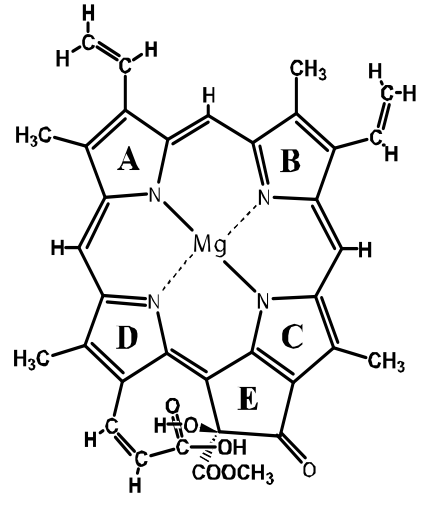

Chl c2'

Figures 21a,b shows the IPCE profiles and the $I-V$ curves, respectively, for solar cells using the set of four sensitizers [10]. The values of $J_{\mathrm{sc}}$ and $\eta$ decrease, all in the order, $\mathrm{Chl} c_{2}>\mathrm{Chl} c_{1}>\mathrm{Chl} c_{2}{ }^{\prime} \geq$ Chl $c_{1}$ '; the $V_{\mathrm{oc}}$ value also decreases in the same order. Interestingly, the decreasing order is in agreement with that of the electron density on the vinyl $\mathrm{H}$, suggested by the H-chemical-shift values, but not necessarily with that of the decreasing order of $E_{\mathrm{ox}}$, i.e., $\mathrm{Chl} c_{1}>\mathrm{Chl} c_{2}>\mathrm{Chl} c_{1}$ ' $>\mathrm{Chl} c_{2}$ '.

Figure 21. (a) The IPCE profiles and (b) the $I-V$ curves of solar cells using the $\mathrm{Chl} c_{1}, \mathrm{Chl}$ $c_{2}, \mathrm{Chl} c_{1}{ }^{\prime}$ and $\mathrm{Chl} c_{2}{ }^{\prime}$ sensitizers (reprinted from [10] with permission of Elsevier).

(a)

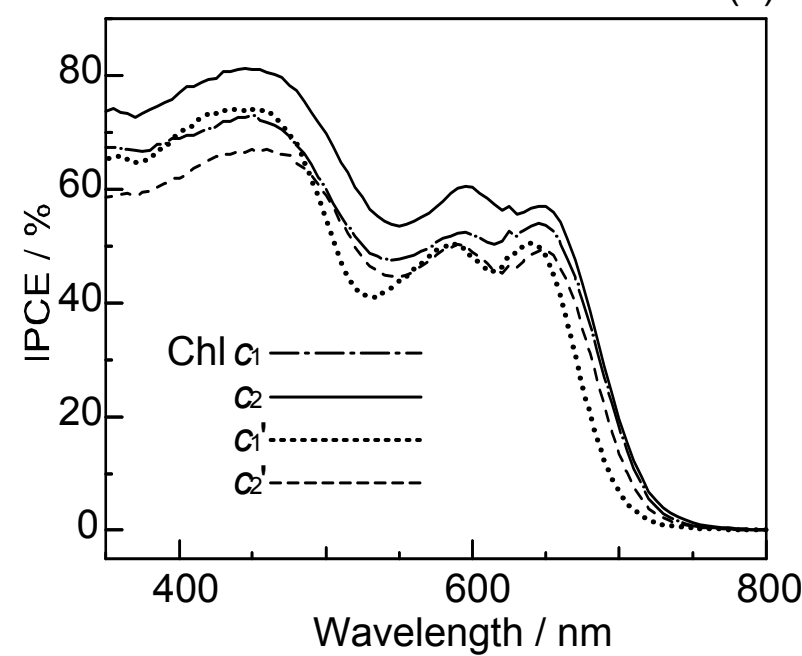

(b)

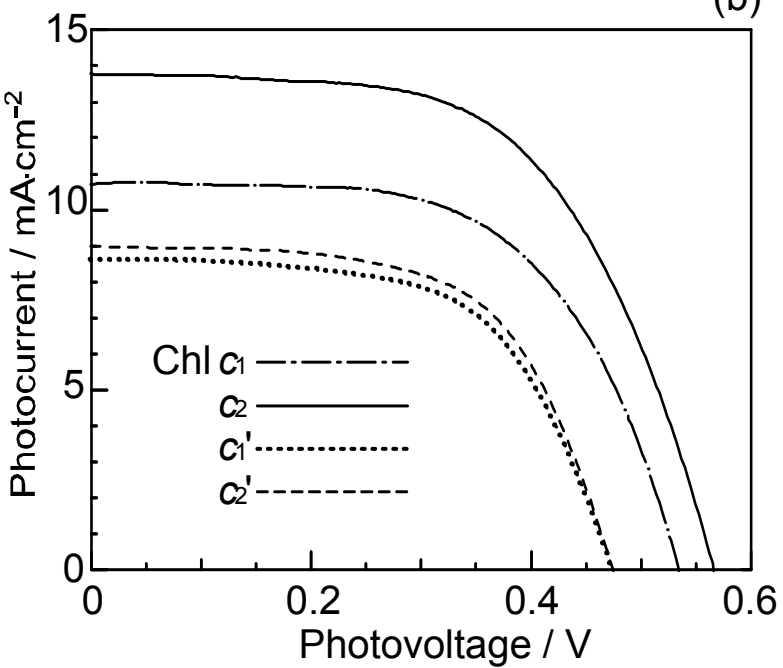

Concerning the Chl $c_{2}$-sensitizerd solar cell, Figure 22a shows that the photocurrent $\left(J_{\mathrm{sc}}\right)$ and conversion efficiency $(\eta)$ monotonously decreased toward the lower dye concentration, whereas Figure $22 \mathrm{~b}$ shows that both the $J_{\mathrm{sc}}$ and $V_{\mathrm{oc}}$ values decreased toward the lower light intensity. There is no sign of singlet-triplet annihilation reaction due to the aggregate formation at all, in this particular sensitizer [10]. 
Figure 22. (a) Concentration dependence and (b) the light-intensity dependence of the $I-V$ curves in Chl $c_{2}$-sensitized solar cell (reprinted from [10] with permission of Elsevier).

(a)

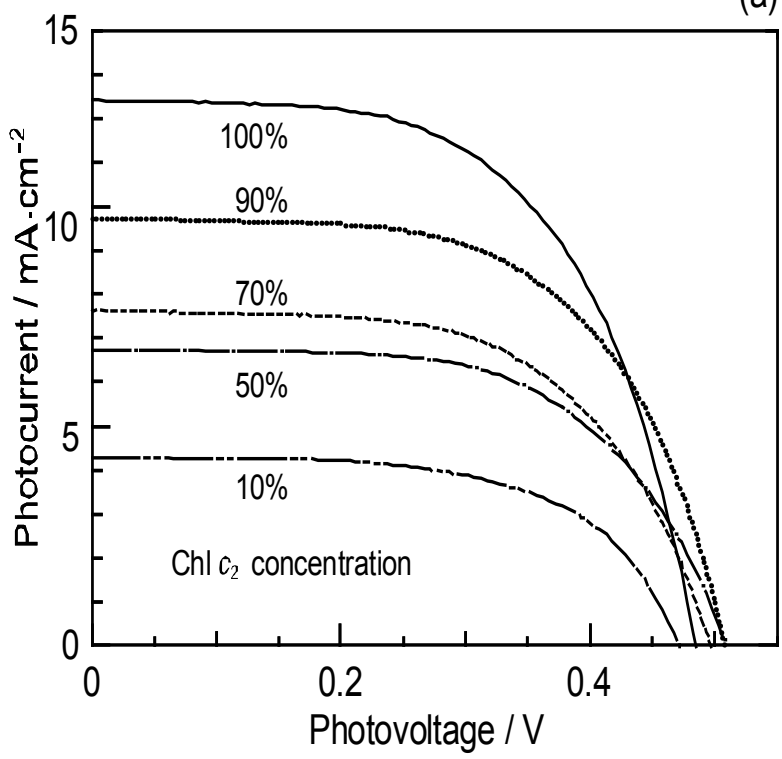

(b)

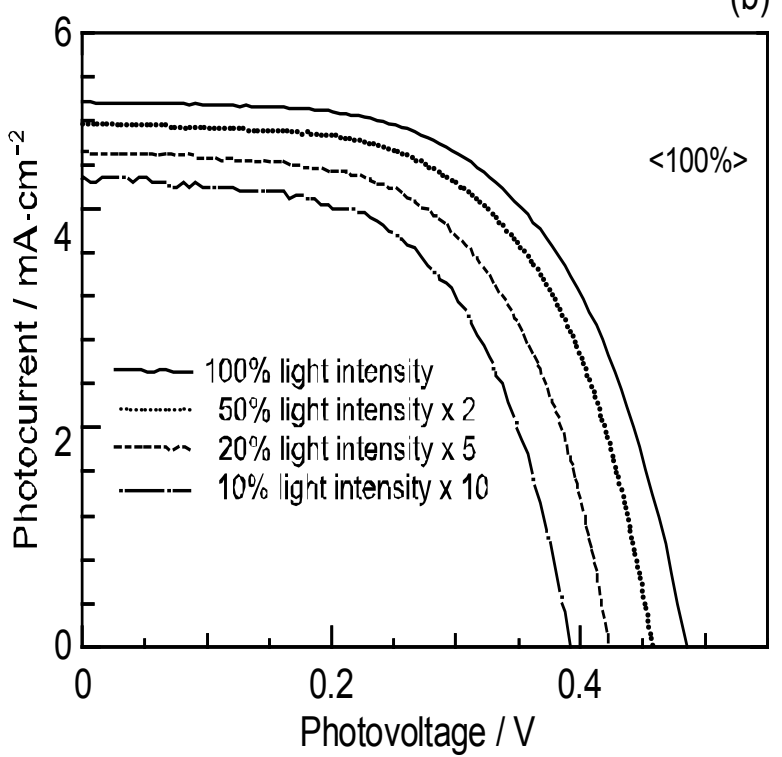

Chl $c_{2}$ (Mg-Phe $\left.c_{2}\right)$ has exhibited the highest photocurrent $\left(J_{\mathrm{sc}}=13.8 \mathrm{~mA} \cdot \mathrm{cm}^{-2}\right)$ and conversion efficiency $(\eta=4.6 \%)$ among all the sensitizers we have tested. It is rather surprising that Phe $c_{2}$ showed one of the lowest photocurrents $\left(J_{\mathrm{sc}}=6.6 \mathrm{~mA} \cdot \mathrm{cm}^{-2}\right)$ and conversion efficiency $(\eta=1.1 \%)$, although their electronic absorption spectra are similar to each other. Most importantly, however, the one-electron oxidation potential of $\mathrm{Chl} c_{2}(1.06 \mathrm{~V})$ is much lower than that of Phe $c_{2}(1.33 \mathrm{eV})$.

\section{Mechanism of Suppression and Enhancement of Photocurrent/Conversion Efficiency by Cosensitization of Pheophorbide Sensitizers without and with Metal, Mg or Zn}

Figure 23 presents the structures of sensitizers used in this section [7]. The structures can be characterized from two different viewpoints: (a) The type of macrocycle. The sensitizers can be classified into three different categories: (i) Phe $a, \mathrm{Mg}$-Phe $a(\mathrm{Chl} a$ ) and Phe $y$, having the chlorin macrocycle like Chl $a$, can be classified into the ' $a$-type' sensitizers. (ii) Phe $b$ consisting of the chlorin macrocycle, to which a pair of $\mathrm{C}=\mathrm{O}$ groups is. (b) The positions of the carboxyl group: The sensitizers can be classified into three different attached in the diagonal positions like Chl $b$ (Mg-Phe $b$ ), can be classified into the ' $b$-type' sensitizer. (iii) $\mathrm{Zn}$-Phe $c_{1}$ and $\mathrm{Mg}$-Phe $c_{2}\left(\mathrm{Chl} c_{2}\right)$ having the porphyrin macrocycle like $\mathrm{Chl} c$, can be classified into the 'c-type' sensitizersgroups in terms of the positions of the carboxyl group. (i) The carboxyl group is directly attached to ring $\mathrm{A}$ in Phe $a$ and $\mathrm{Mg}$-Phe $a$, but through an additional double bond in Phe $y$, (ii) it is attached to ring $\mathrm{B}$ in Phe $b$, and (iii) it is attached to ring $\mathrm{D}$ through a double bond in $\mathrm{Zn}$-Phe $c_{1}$ and $\mathrm{Mg}$-Phe $c_{2}\left(\mathrm{Chl} c_{2}\right)$. In terms of the $x$-axis and the $y$-axis that have been originally defined for the $\mathrm{Q}_{x}$ and $\mathrm{Q}_{y}$ transitions of $\mathrm{Chl} a$, the carboxyl group is on the $y$-axis in Phe $a$, Mg-Phe $a$ and Phe $y$, whereas it is on the $x$-axis in Phe $b, \mathrm{Zn}$-Phe $c_{1}$ and Mg-Phe $c_{2}$. See the sticks of arrows directing to the carboxyl group, which can be classified into two directions. 
Figure 23. Chemical structures of Phe sensitizers without and with metal, $\mathrm{Mg}$ or $\mathrm{Zn}$ (reprinted from [7] with permission of MDPI Publishing).

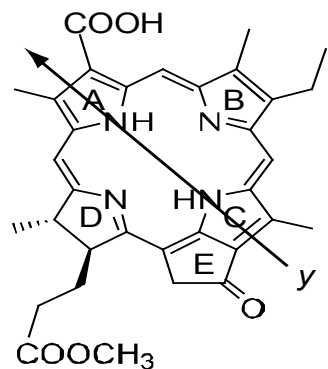

Phe a

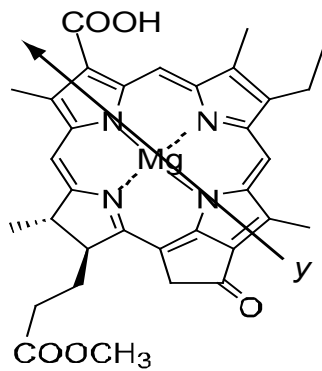

Mg-Phe a (Chl a ) a-type

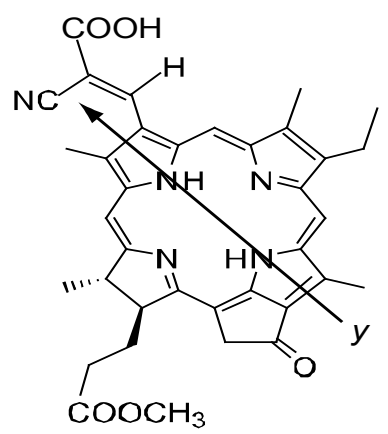

Phe $y$

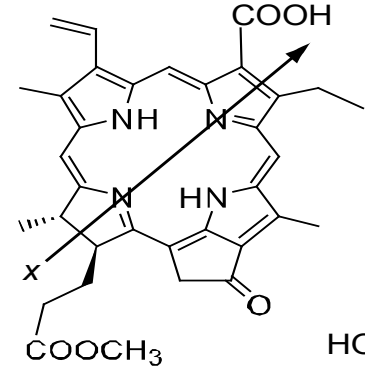

Phe $b$

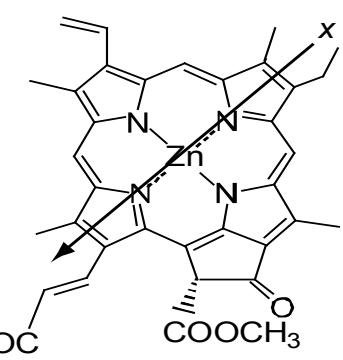

$\mathrm{Zn}-\mathrm{Chl} c_{1}$

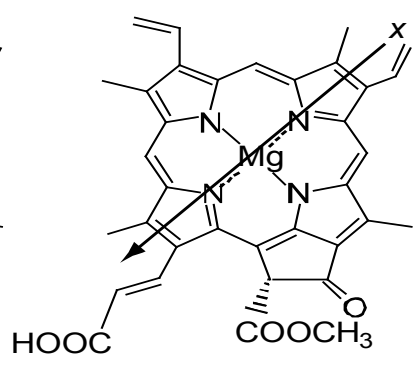

Mg-Phe $c_{2}(\mathrm{Chl} \mathrm{c2})$

$b$-type

Figure 24 exhibits (a) the IPCE profiles and (b) the $I-V$ curves for the five pairs of sensitizers, which can be classified into three different types of cosensitization, i.e., $a$-type $+a$-type, $a$-type + $b$-type and $a$-type $+c$-type.

Figure 24. (a) The IPCE profiles for three different types of co-sensitization and (b) the $I-V$ curves for three different types of co-sensitization.
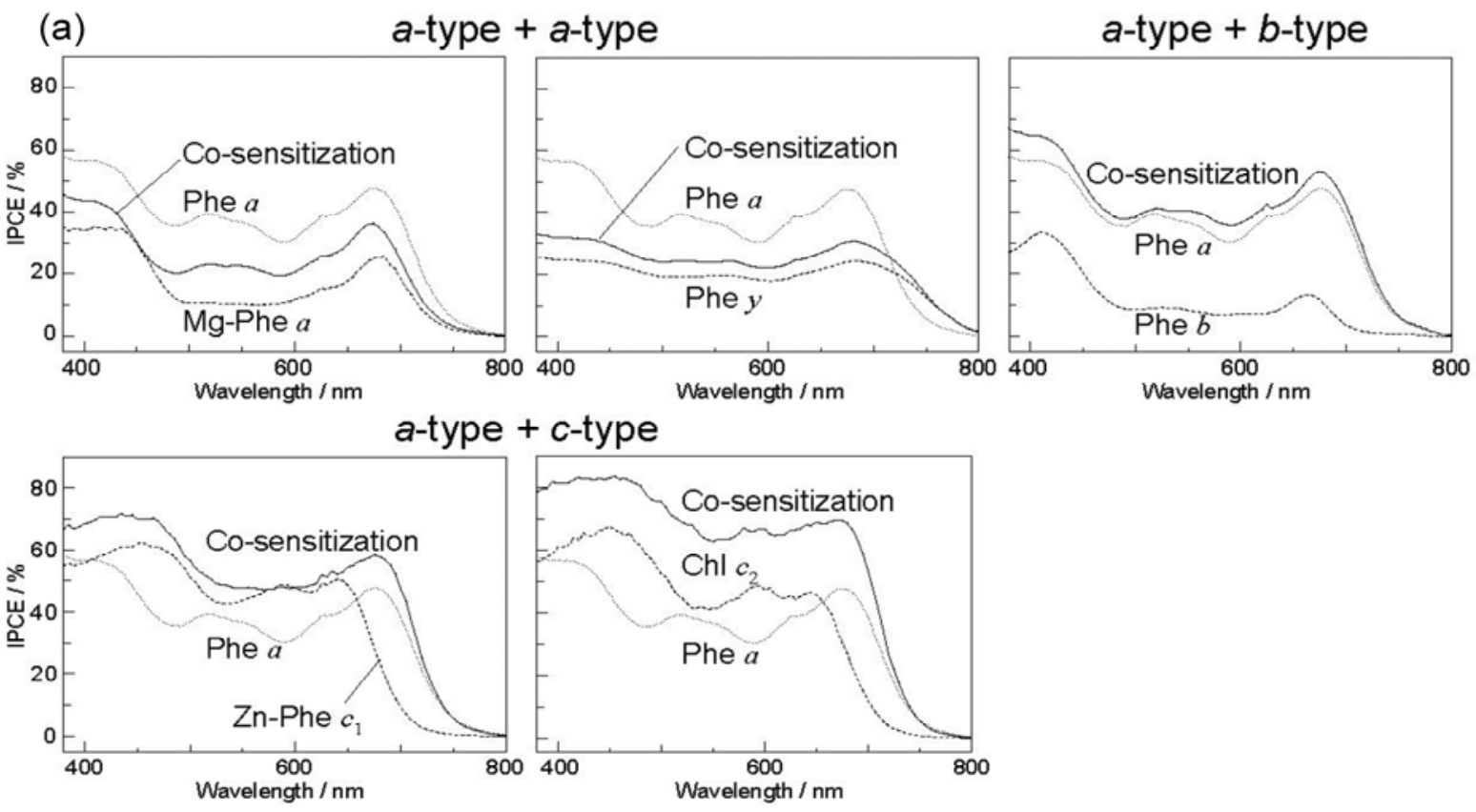
Figure 24. Cont.
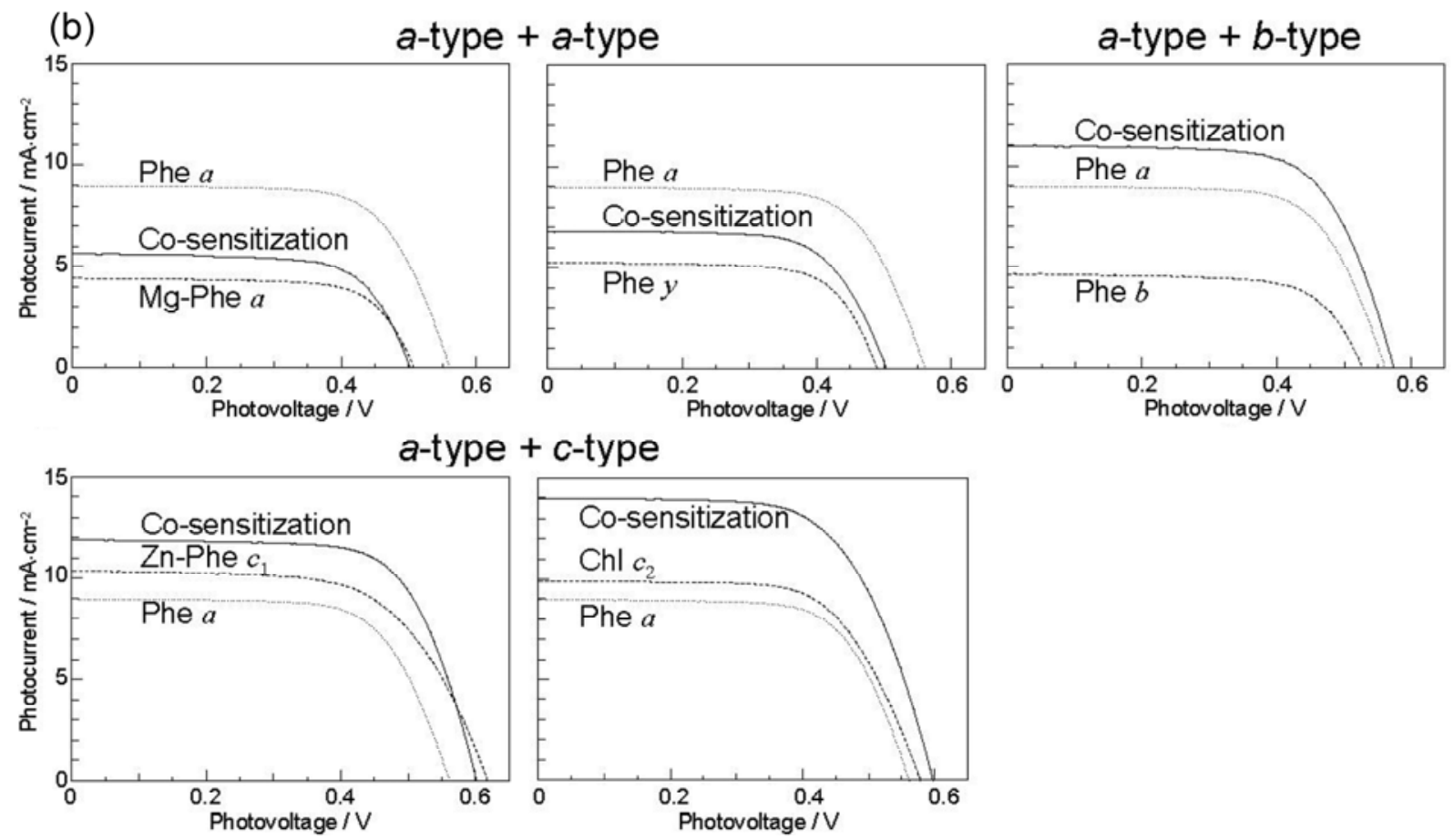

In the present experiments of cosensitization, Phe $a$ was used as the principal sensitizer in common. The IPCE profiles and the $I-V$ curves pictorially demonstrate that the cosensitization (shown in solid line) of $a$-type $+a$-type gives rise to the suppression, whereas those of $a$-type $+b$-type and $a$-type + $c$-type give rise to the enhancement of photocurrent/conversion efficiency.

Table 4 lists the $V_{\mathrm{oc}}, F F, J_{\mathrm{sc}}$ and $\eta$ values for the singly-sensitized solar cells and the $E_{\mathrm{ox}}$ values for the principal and individual cosensitizers (see the captions for their definitions of abbreviated parameters). In comparison to the principal sensitizer, the $J_{\text {sc }}$ and $\eta$ values are smaller in $a$-type and $b$-type cosensitizers, but they are larger in $c$-type cosensitizer. The $V_{\text {oc }}$ and $F F$ values are more or less similar among the set of sensitizers.

Table 4. The open-circuit photovoltage $\left(V_{\text {oc }}\right)$, fill factor $(F F)$, short-circuit photo-current density $\left(J_{\mathrm{sc}}\right)$, conversion efficiency $(\eta)$ of the singly-sensitized solar cells and the one-electron oxidation potential $\left(E_{\mathrm{ox}}\right)$ of each sensitizer.

\begin{tabular}{|c|c|c|c|c|c|}
\hline $\begin{array}{l}\text { The standard and } \\
\text { co-sensitizer }\end{array}$ & $V_{o c} / \mathrm{V}$ & $F F$ & $J_{s c} / \mathbf{m A} \cdot \mathbf{c m}^{-2}$ & $\eta$ & $E_{o x} / \mathrm{V} v s$. NHE \\
\hline Phe $a$ (a-type) & 0.56 & 0.68 & 9.0 & 3.4 & 1.16 \\
\hline \multicolumn{6}{|l|}{ a-type } \\
\hline Mg-Phe $a$ & 0.51 & 0.70 & 4.4 & 1.6 & 0.79 \\
\hline Phe $y$ & 0.49 & 0.70 & 5.2 & 1.8 & 1.19 \\
\hline \multicolumn{6}{|l|}{ b-type } \\
\hline Phe $b$ & 0.53 & 0.70 & 4.6 & 1.7 & 1.24 \\
\hline \multicolumn{6}{|l|}{ c-type } \\
\hline Zn-Phe $c_{1}$ & 0.62 & 0.63 & 10.4 & 4.0 & 1.16 \\
\hline $\mathrm{Mg}$-Phe $c_{2}\left(\mathrm{Chl} c_{2}\right)$ & 0.58 & 0.66 & 13.8 & 4.6 & 1.06 \\
\hline
\end{tabular}


Table 5 lists the $V_{\text {oc }}, F F, J_{\text {sc }}$ and $\eta$ values for the cosensitized solar cells using $a$-type, $b$-type and $c$-type as the cosensitizers. Cosensitization of the principal sensitizer with $a$-type sensitizers give rise to lower $J_{\text {sc }}$ and $\eta$ values, whereas cosensitization with $b$-type and $c$-type sensitizers, give rise to definitively higher $J_{\text {sc }}$ and $\eta$ values. The enhancement factors ${ }^{\mathrm{r}} J_{\mathrm{sc}}$ and ${ }^{\mathrm{r}} \eta$ (defined below the table) are definitely higher (lower) in the latter (former) cosensitizations. Spectral separation, $S$, is also listed in the last column with its definition at the bottom.

Table 5. The open-circuit photovoltage $\left(V_{\text {oc }}\right)$, fill factor $(F F)$, short-circuit photo-current density $\left(J_{\mathrm{sc}}\right)$, conversion efficiency $(\eta)$ of the solar cells co-sensitized, and the spectral separation (S). The definitions of ${ }^{\mathrm{r}} J_{\mathrm{sc}}$ and ${ }^{\mathrm{r}} \eta$ are given below the table.

\begin{tabular}{|c|c|c|c|c|c|c|c|c|}
\hline Co-sensitizers & $V_{o c} / \mathbf{V}$ & $F F$ & $J_{\mathrm{sc}} / \mathrm{mA} \cdot \mathrm{cm}^{-2}$ & ${ }^{\mathrm{r}} J_{\text {sc }}$ & $\eta$ & ${ }^{\mathrm{r}} \boldsymbol{\eta}$ & $\frac{{ }^{r} J_{s c}+{ }^{r} \boldsymbol{\eta}}{2}$ & $S$ \\
\hline \multicolumn{9}{|l|}{ a-type } \\
\hline Mg-Phe $a$ & 0.50 & 0.69 & 5.6 & 0.83 & 1.9 & 0.76 & 0.8 & 41 \\
\hline Phe $y$ & 0.50 & 0.68 & 6.8 & 0.97 & 2.3 & 0.88 & 0.9 & 62 \\
\hline \multicolumn{9}{|l|}{ b-type } \\
\hline Phe $b$ & 0.57 & 0.68 & 10.9 & 1.60 & 4.3 & 1.65 & 1.6 & 39 \\
\hline \multicolumn{9}{|l|}{ c-type } \\
\hline Zn-Phe $c_{1}$ & 0.60 & 0.69 & 11.9 & 1.23 & 5.0 & 1.35 & 1.3 & 80 \\
\hline Mg-Phe $c_{2}\left(\mathrm{Chl} c_{2}\right)$ & 0.60 & 0.64 & 14.0 & 1.47 & 5.4 & 1.50 & 1.5 & 95 \\
\hline
\end{tabular}

Concerning cosensitization, the three different pairs of sensitizers give rise to suppression or enhancement in reference to the average of performance of the component sensitizers: (i) The $a$-type + $a$-type cosensitization gives rise to suppression of performance; the relative performance values decrease for both sensitizers, i.e., Mg-Phe $a\left({ }^{r} J_{\mathrm{sc}}=0.83,{ }^{r} \eta=0.76\right)$ and Phe $y\left({ }^{r} J_{\mathrm{sc}}=0.97,{ }^{r} \eta=0.88\right)$, the averaged ratios being $\sim 0.8$ and $\sim 0.9$, respectively. (ii) The $a$-type $+b$-type cosensitization with the cosensitizer, Phe $b$, shows remarkably-high enhancement $\left({ }^{r} J_{\mathrm{sc}}=1.60,{ }^{r} \eta=1.65\right)$, the averaged ratio being 1.6. (iii) The $a$-type $+c$-type cosensitization causes large enhancement with the sensitizers, Zn-Phe $c_{1}\left({ }^{r} J_{\mathrm{sc}}=1.23,{ }^{r} \eta=1.35\right)$ and Mg-Phe $c_{2}\left({ }^{r} J_{\mathrm{sc}}=1.47,{ }^{r} \eta=1.50\right)$, the averaged ratio being $\sim 1.3$ and $\sim 1.5$, respectively. Importantly, the combination of the chlorin (Phe $a$ ) and the porphyrin (Mg-Phe $c_{2}$ ) sensitizers, each showing the highest two individual performance, gave rise to the highest enhancement of the $J_{\mathrm{sc}}$ value $\left(9.0\right.$ and $\left.13.8 \rightarrow 14.0 \mathrm{~mA} \cdot \mathrm{cm}^{-2}\right)$ and the $\eta$ value $(3.4$ and $4.6 \rightarrow 5.4 \%)$.

Figure 25 shows the electronic-absorption spectra of the pairs of sensitizers in ethanol solution [7], which can be characterized as follows: Individual sensitizers: Chlorin sensitizers of both $a$-type (Mg-Phe $a$ and Phe $y$ ) and $b$-type (Phe $b$ ) clearly exhibit the Soret, $\mathrm{Q}_{x}$ and $\mathrm{Q}_{y}$ absorption peaks, whereas the metal-porphyrin sensitizers of $c$-type (Zn-Phe $c_{1} \& \mathrm{Mg}$-Phe $c_{2}$ ) exhibit the Soret peak on the longer-wavelength side and a pair of peaks (possibly $\mathrm{Q}_{x}$ and $\mathrm{Q}_{y}$ ) on the shorter-wavelength side. $A$ pair of cosensitizers: Depending on the overlapped and split absorption peaks due to the pair of sensitizers, competitive or complementary light absorption is expected to take place. Concerning the overlap of cosensitizer absorption peaks, (i) the ' $a$-type $+a$-type' cosensitizer pair and the ' $a$-type $+b$-type' pair are overlapped in a complicated ways. However, (ii) the ' $a$-type $+c$-type' pair exhibits no 
overlaps in either the Soret or the $\mathrm{Q}_{y}$ absorptions. To evaluate the overlap over the spectral region, we have defined 'spectral separation $(S)$ ':

$$
S=\int\left|\varepsilon_{A}(\lambda)-\varepsilon_{B}(\lambda)\right| d \lambda
$$

and the values are listed in Table 5 (right end). Importantly, it is rather small in the $a$-type $+a$-type and $a$-type $+b$-type pairs and the largest in the $a$-type $+c$-type pairs.

Figure 25. The electronic absorption spectra of the pairs of co-sensitizers in ethanol solution. (a) Phe $a+\mathrm{Mg}$-Phe $a$; (b) Phe $a+$ Phe $y$; (c) Phe $a+$ Phe $b$; (d) Phe $a+\mathrm{Zn}$-Phe $c_{1}$ and (e) Phe $a+$ Mg-Phe $c_{2}$ (reproduced with permission from [7] of MDPI Publishing).
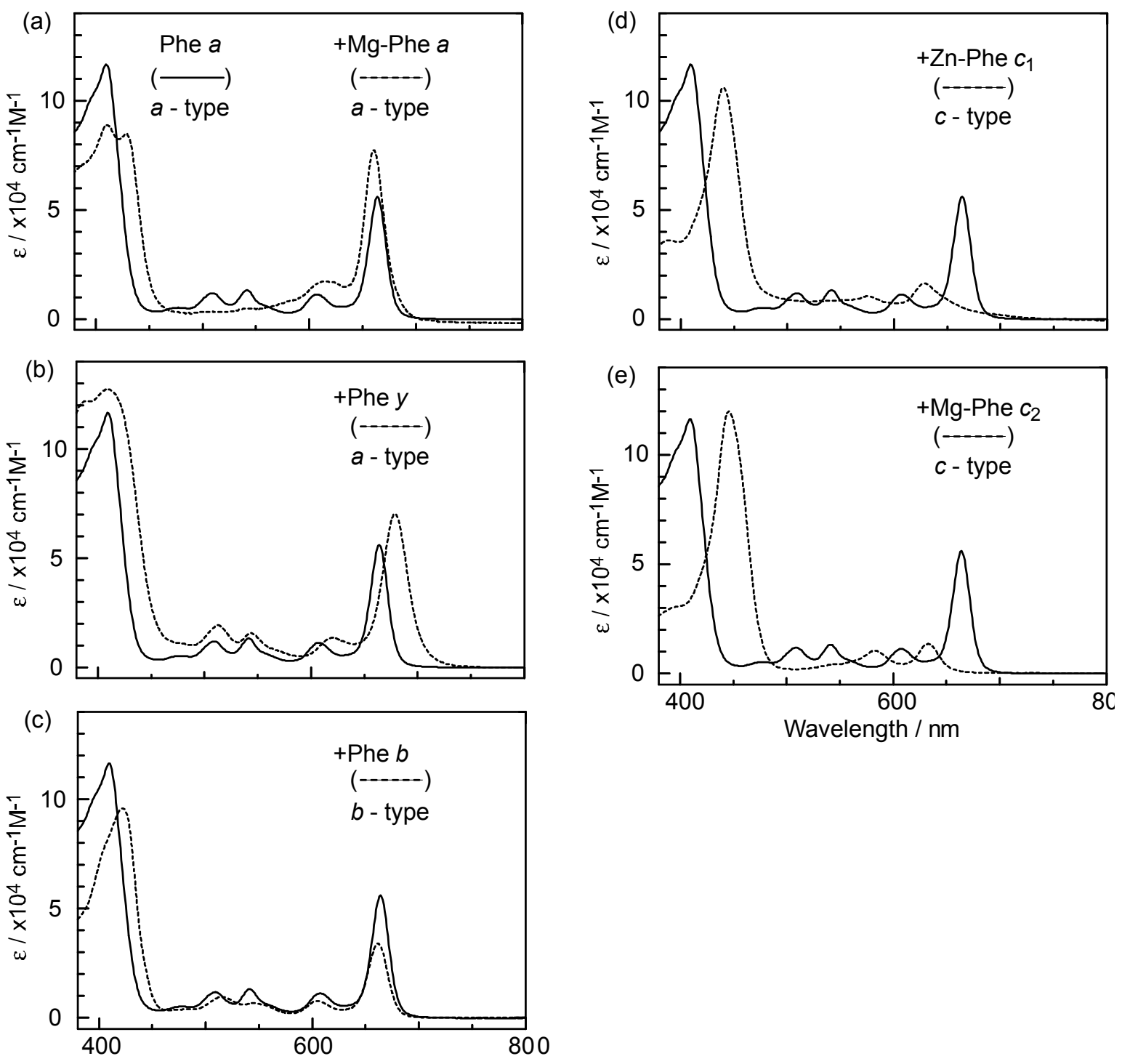

We examined the effects due to the type of macrocycles and the position of the carboxyl group on the molecular orbitals by means of the time-dependent density-function-theory (TD-DFT) calculations: Figure 26 shows the calculated four major molecular orbitals, including HOMO-1, HOMO, LUMO and LUMO+1 (here, HOMO and LUMO stands for the highest-occupied molecular orbital and the lowest-unoccupied molecular orbital, respectively). 
Figure 26. The four orbitals including HOMO-1, HOMO, LUMO and LUMO+1 obtained by TD-DFT calculations (reproduced from [7] with permission of MDPI Publishing).

(a) Phe a

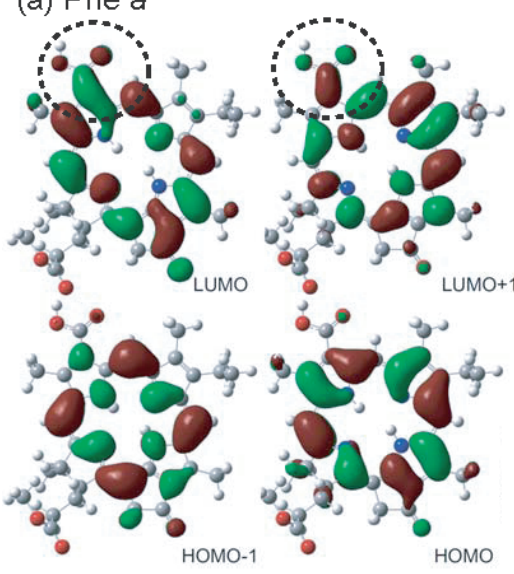

(d) Phe $b$
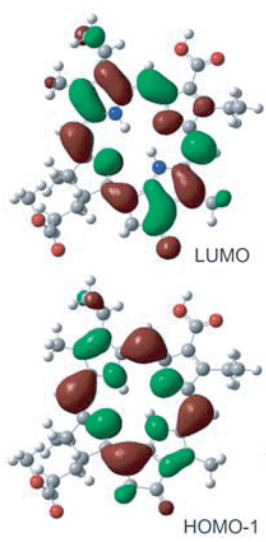
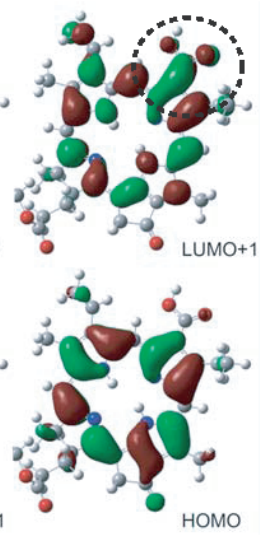

(b) Mg-Phe a
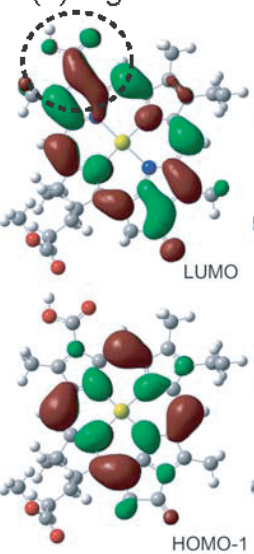

(e) Zn-Phe $c_{1}$
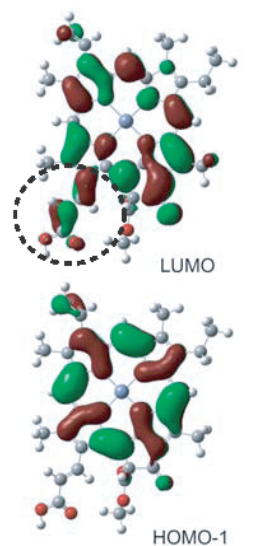

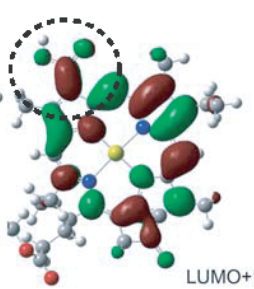

(2)

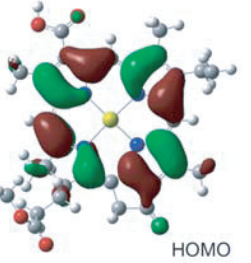

номо
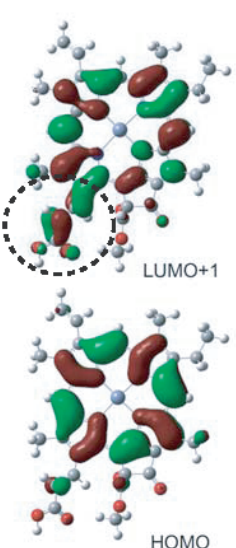

(c) Phe $y$

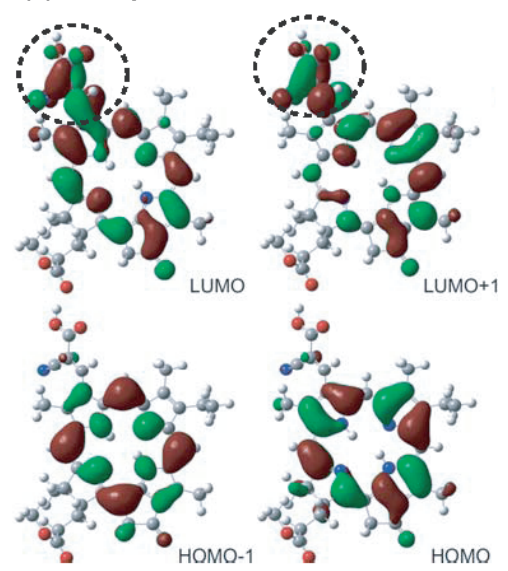

(f) $\mathrm{Mg}-\mathrm{Phe} \mathrm{c}_{2}$
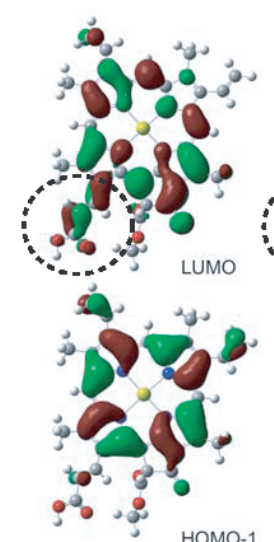
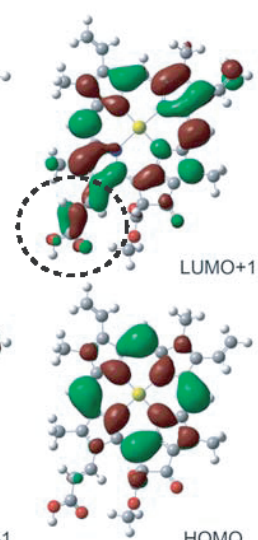

The shapes of the four molecular orbitals are different depending on the type of macrocycle, chlorin or porphyrin. The LUMO and LUMO+1, that are expected to play the key role in the electron injection into $\mathrm{TiO}_{2}$, are found to be extended toward the carboxyl group; in other words, the electron density is shifted toward the carboxyl group to get ready for electron injection (see the regions shown in dotted circles). Also, the electronic transitions are mainly determined by the combination of the (HOMO-1, $\mathrm{HOMO}\} \rightarrow\{\mathrm{LUMO}, \mathrm{LUMO}+1\}$ transitions and, therefore, all the Soret, $\mathrm{Q}_{x}$ and $\mathrm{Q}_{y}$ transitions are expected to be strongly influenced by the position of the carboxyl group (or, in other words, by the direction of polarization).

The results of DFT calculations shown in Figure 26 [7] have provided us with a strong support to the ideas that the type of macrocycle, chlorin or porphyrin, and the position of the carboxyl group, on the $y$-axis or the $x$-axis, strongly affect the directions of electron-injection and transition-dipole moment.

The suppression or enhancement of performance in cosensitization can be explained in terms of the light absorption (competitive or complementary), the direction of transition-dipole moment (parallel or orthogonal) and the singlet-energy transfer (interactive or independent) between the pair of sensitizers:

(i) The absorption spectra of the sensitizers (in Figure 25) show that the major light absorptions are absolutely complementary in the $a$-type $+c$-type pair. Therefore, the highest enhancement in the 
$a$-type $+c$-type cosensitization can be rationalized in terms of complementary absorption not only in the $\mathrm{Q}_{x}$ and $\mathrm{Q}_{y}$ levels but also in the Soret levels.

(ii) The combination of the $a$-type sensitizer having the carboxyl group in the $y$-direction and the $b$-type or $c$-type sensitizer having the carboxyl group in the $x$-direction can give rise to the highest enhancement of photocurrent and conversion efficiency, because of the minimum interference of the transition dipoles between the pair of cosensitizers. Polarization and electron-injection along the orthogonal directions must prevent the interference between the intermolecular transition dipoletransition dipole interactions that can trigger intermolecular energy transfer and the resultant dissipation of the singlet energy.

(iii) A pair of electron injections through energetically different levels, i.e., one, the lower level of the $\mathrm{Q}_{y}$ absorption of Phe $a$ and the other, the higher level of the Soret absorption of Chl $c_{2}\left(\mathrm{Mg}-\mathrm{Phe} c_{2}\right)$ is expected to have little interference between each other (see Figure 27 [7]).

Figure 27. Effects of the one-electron oxidation potential and the pattern on the electroninjection channels through the tunneling mechanism. No strong correlation between the electron injections from the Phe $a$ and $\mathrm{Chl} c_{2}$ sensitizers are expected (reproduced from [7] with permission of MDPI Publishing).

Phe a

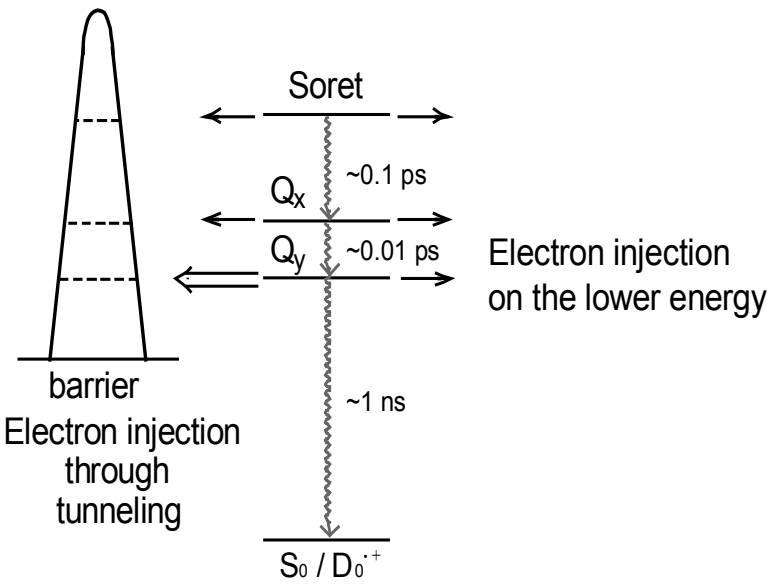

Higher $E_{\mathrm{ox}}$

(Lower energy)
Chl $c 2$

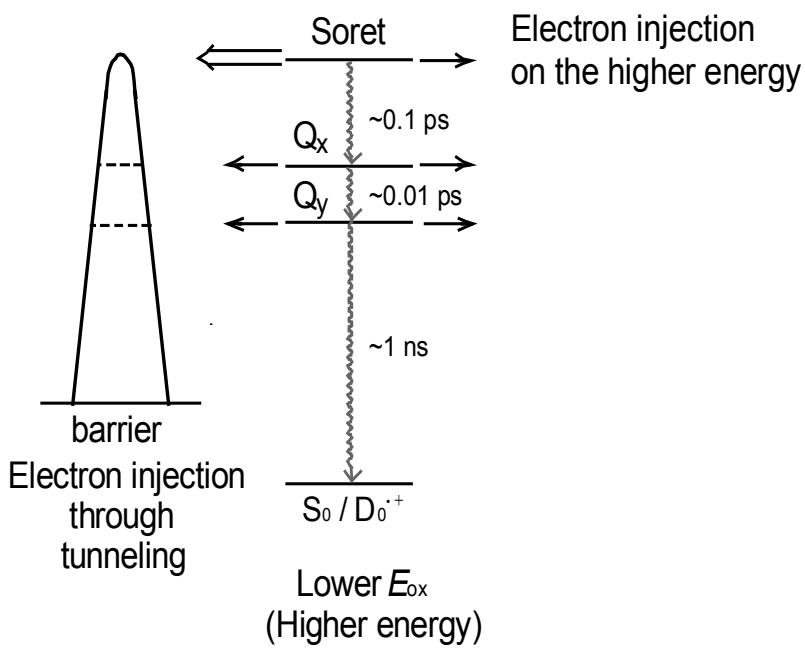

Cosensitization by the use of the best and the second-best sensitizers, i.e., Chl $c_{2}$ (Mg-Phe $c_{2}$ ) and Phe $a$, we have achieved the maximum enhancement in photocurrent $\left(J_{\mathrm{sc}}=14.0 \mathrm{~mA} \cdot \mathrm{cm}^{-2}\right)$ and conversion efficiency $(\eta=5.4 \%)$, the enhancement factor being 1.47 and 1.50 times in reference to the averaged value of the performance of the component cosensitizers. The enhancement is ascribed to the complementary light absorption, the orthogonal transition-dipole moments and the energetically different pathways of electron injection. 


\section{Regression: Singlet-Triplet Annihilation Reaction}

We have provided specialized readers with enough information, extracted from the raw data, concerning the mechanisms of suppression and enhancement of photocurrent/conversion efficiency (performance) in dye-sensitized solar cells using carotenoid and chlorophyll derivatives as sensitizers (the title of this review). However, it might be worthwhile, for non-specialized readers, to add 'a regression section' concerning one of the most important subjects in this review, i.e., 'singlet-triplet annihilation', by referring to and correlating Figures and Tables according to the order of the previous sections and subsections, after an easy Introduction. Those readers who are interested are encouraged to read the previous sections $1-3$ again according to our guide.

\subsection{An Easy Introduction to Mechanisms of Singlet-Triplet Annihilation}

There are two different kinds of electronic states, singlet and triplet, with anti-parallel and parallel pairs of spins. The names originate from their number of splittings, when placed under the external magnetic field. The most stable state is the ground state $\left(\mathrm{S}_{0}\right)$, where a pair of anti-parallel spins is present on the same lowest electronic level. When one of the spins goes to the next upper state, it becomes the singlet excited-state $\left(S_{1}\right)$. When the inversion of the upper spin takes place to form a parallel spin, it becomes the lower triplet state $\left(T_{1}\right)$. Then, the overall state is $S_{1}+T_{1}$, from which the 'singlet-triplet annihilation' reaction starts, as shown in Figure 28.

Figure 28. Mechanism of singlet-triplet annihilation. When a pair of the $S_{1}$ and $T_{1}$ states becomes close-by, a resonance electron transfer takes place, one downward and the other upward. As a result, the $S_{1}$ singlet-excited state becomes annihilated and the $T_{1}$ state becomes excited to an upper $\mathrm{T}_{n}$ triplet state (the left $\rightarrow$ middle transformation; an overall $\mathrm{S}_{1}+\mathrm{T}_{n}$ state is generated). Then, the $\mathrm{T}_{n}$ state quickly relaxes down to the $\mathrm{T}_{1}$ state, because the inversion of spin is unnecessary (the middle $\rightarrow$ right transformation; an overall $\mathrm{S}_{0}+\mathrm{T}_{1}$ state resulted). Through this two-steps of reaction, the $\mathrm{S}_{1} \rightarrow \mathrm{S}_{0}$ singlet transformation is mediated by the $\mathrm{T}_{1}$ state, which is called 'singlet-triplet annihilation'.

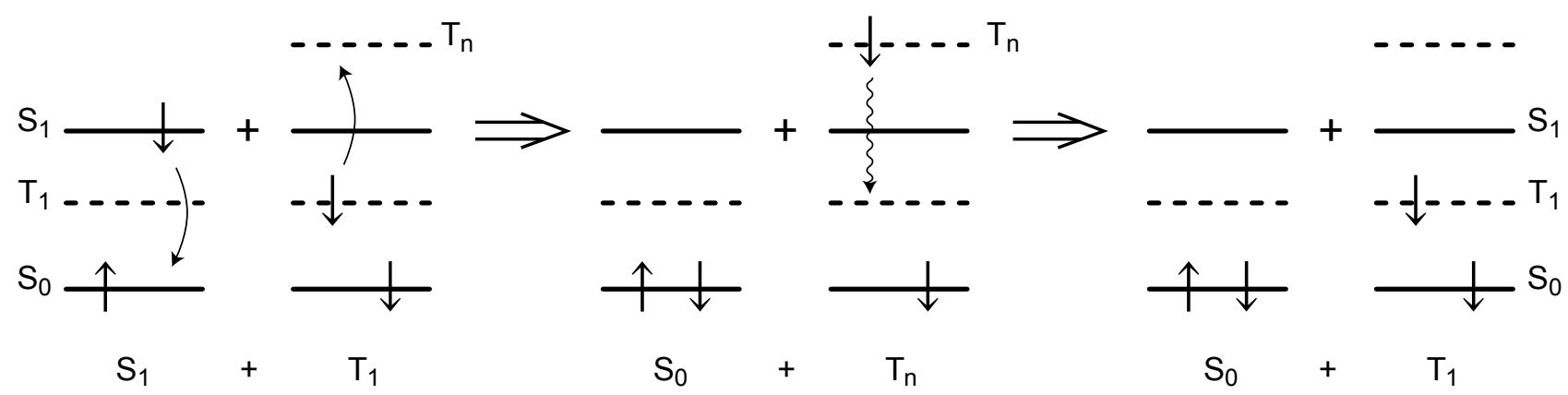

When the $S_{1}$ and $T_{1}$ states become close-by, a resonance electron transfer takes place, one downward and the other upward. As a result, the singlet-excited state $\left(\mathrm{S}_{1}\right)$ is annihilated and the $\mathrm{T}_{1}$ state becomes excited to an upper state $\left(\mathrm{T}_{n}\right)$ (as shown in the left end of the figure). Then, the $\mathrm{T}_{n}$ state can quickly relaxes down to the $\mathrm{T}_{1}$ state, because the inversion of spin is unnecessary (as shown in the middle). As an overall reaction, the $S_{1}$ excitation, which can inject the upper electron to a 
semiconductor $\mathrm{TiO}_{2}$ in our solar cells, becomes annihilated (as shown in the right-end). The resultant $\mathrm{T}_{1}$ state is now ready to annihilate a next $\mathrm{S}_{1}$ excitation.

The most important difference between the $S_{1}$ and $T_{1}$ states is their lifetimes. The $S_{1}$ state can relax down to the $S_{0}$ state instantaneously because no inversion of spin is necessary, whereas the $T_{1}$ state takes $\sim 10^{4}$ longer time to relax down to the $\mathrm{S}_{0}$ state, because the inversion of the upper spin is necessary under the influence of the external magnetic field.

This 'single-triplet annihilation' reaction can take place, only when the pair of the $S_{1}$ and $T_{1}$ excitations becomes close-by. When the interaction between them is not strong enough or sterically hindered, the singlet-triplet annihilation reaction can never take place.

\subsection{A Brief Summary of Sections 1-4, Focusing on the Singlet-Triplet Annihilation Reaction}

In Section 1, the conjugation-length dependence in photocurrent/conversion efficiency (performance) of DSSCs, using RA and CAs sensitizers (Figure 1), was determined by the use of $I-V$ curves (Figure 2). The performance was the highest in CA7, and declined toward both RA5 and CA13 (Figures 3a,b). The latter decrease was nicely explained in terms of the pathways and time constants of electron injection immediately after excitation, i.e., $<10$ ps, for CA7-CA11 (Figure 5). The electroninjection efficiencies are listed in Table 1 and presented in Figure 3c. However, the decline toward RA5 (Figure 3a,b) was left to be explained.

This decline toward the shorter chain was explained by analyzing the later stages after excitation, i.e., $<40 \mu \mathrm{s}$, as presented in Figure 6 and Table 3. It was found that a combined $\mathrm{D}_{0}{ }^{\bullet+}+\mathrm{T}_{1}$ state split into a mixture of the $\mathrm{D}_{0}{ }^{-+}$state and $\mathrm{T}_{1}$ state (Figure 6). Here, the $\mathrm{T}_{1} / \mathrm{D}_{0}{ }^{\bullet+}$ ratio was similar between the combined state and the split states, which indicates that the $\mathrm{T}_{1} / \mathrm{D}_{0}{ }^{\bullet+}$ ratio is determined by the energy gap between the conduction band edge (CBE) and the $T_{1}$ state shown in Figure 7.

It is important that the yield of the $\mathrm{D}_{0}{ }^{\cdot+}$ state generated by electron injection decreased, whereas that of the $\mathrm{T}_{1}$ state increased systematically in the order, CA8 $\rightarrow$ CA7 $\rightarrow$ CA6 $\rightarrow$ RA5 bound to $\mathrm{TiO}_{2}$ (Table 3).

In Section 2, we tried to obtain more direct evidence for the presence of singlet-triplet annihilation: In Subsection 2.1, the dependence of performance on the dye concentration in CA7-sensitized solar cell is presented. A unique concentration dependence is seen in the $I-V$ curves shown in Figure 9. The photocurrent, $J_{\mathrm{sc}}$, and the conversion efficiency, $\eta$, are plotted as the functions of the CA7 concentration in Figure 10. The highest performance was seen at $70 \%$ and the second-highest, at 30\%; both were higher than the value at $100 \%$.

In Figure 10, key changes were characterized in terms of (i) coherent, completely delocalized excitation at $100 \%$, (ii) partially-destroyed delocalized excitation at $90 \%$, (iii) localized migrating excitation at $70 \%$, and (iv) a maximum isolated excitation at $30 \%$. The typical arrangement of the dye $(\circ)$ and the spacer $(\bullet)$ molecules depicted in Figure 11 was used for this explanation.

In Subsection 2.2, much more convincing evidence for the singlet-triplet annihilation reaction was obtained by the use of a set of medium-sized conjugated CAs having different transition-dipole moments (Figure 12). (a) The concentration dependence and (b) the light-intensity dependence of the photocurrent (presented in Figure 13) and the conversion efficiency (Figure 14) showed the following 
trends in the least-polarizable dye sensitizer, i.e., $\phi-6-\mathrm{CA}$. Both the photocurrent and conversion efficiency decreased toward the lower dye concentration or the lower light intensity, as we expect.

On the other hand, both the photocurrent and the conversion efficiency increased toward the lower dye concentration and the lower light intensity, in the most-polarizable dye sensitizer, i.e., $\mathrm{Me}_{2} \mathrm{~N}-\phi$ $\mathrm{CA}$, contrary to our expectation. Concerning the latter, the two different concentrations of the dye sensitizer were used. This observation can be explained only in terms of 'singlet-triplet annihilation reaction' that is most efficient at the higher dye concentration or at the higher light intensity.

In Section 3, the first type of dependence, as we expect, can be used to show the absence of singlet-triplet annihilation. In Subsection 3.1, it was shown that no singlet-triplet annihilation took place (as shown in Figure 18) due to the steric hindrance of the straight CA sidechain of the Phe-Car adduct sensitizer (Figure 19). In Subsection 3.2, no sign of singlet-triplet annihilation took place, either (Figure 22), due to the rigid and flat porphyrin macrocycle in the $\mathrm{Chl} c_{2}$ sensitizer (Figure 20).

Thus, the absence (or presence) of the dependence on the dye concentration or the light intensity, contrary to our expectation, can be used to determine the absence (or presence) of the singlet-triplet annihilation reaction.

\section{Acknowledgements}

The authors acknowledge permission by MDPI Publishing, RSC Publishing, Elsevier and the American Chemical Society to use in this article schemes, figures and tables which appeared in our previous papers.

\section{References}

1. Koyama, Y.; Rondonuwu, F.S.; Fujii, R.; Watanabe, Y. Light-harvesting function of carotenoids in photo-synthesis: The roles of the newly found $1{ }^{1} \mathrm{~B}_{\mathrm{u}}{ }^{-}$state. Biopolymers 2004, 74, 2-18.

2. Koyama, Y.; Kakitani, Y.; Watanabe, Y. Photophysical properties and light-harvesting and photoprotective functions of carotenoids in bacterial photosynthesis: Structural selections. In Primary Processes of Photosynthesis, Part 1: Principles and Apparatus (Comprehensive Series in Photochemical and Photobiology volume 8), Renger, G., Ed.; RSC Publishing: Cambridge, UK, 2008; pp. 151-201.

3. Wang, X.-F.; Fujii, R.; Ito, S.; Koyama, Y.; Yamano, Y.; Ito, M.; Kitamura, T.; Yanagida, S. Dye-sensitized solar cells using retinoic acid and carotenoic acids: Dependence of performance on the conjugation length and the dye concentration. Chem. Phys. Lett. 2005, 416, 1-6.

4. Xiang, J.; Rondonuwu, F.S.; Kakitani, Y.; Fujii, R.; Watanabe, Y.; Koyama, Y.; Nagae, H.; Yamano, Y.; Ito, M. Mechanisms of electron injection from retinoic acid and carotenoic acids to $\mathrm{TiO}_{2}$ nanoparticles and charge recombination via the $\mathrm{T}_{1}$ state as determined by subpicosecond to microsecond time-resolved absorption spectroscopy: Dependence on the conjugation length. J. Phys. Chem. B 2005, 109, 17066-17077.

5. Furuichi, K.; Sashima, T.; Koyama, Y. The first detection of the $3 \mathrm{~A}_{\mathrm{g}}{ }^{-}$state in carotenoids using resonance-Raman excitation profiles. Chem. Phys. Lett. 2002, 356, 547-555. 
6. Fujii, R.; Inaba, T.; Watanabe, Y.; Koyama, Y.; Zhang, J.-P. Two different pathways of internal conversion in carotenoids depending on the length of the conjugated chain. Chem. Phys. Lett. 2003, 369, 165-172.

7. Koyama, Y.; Miki, T.; Wang, X.-F.; Nagae, H. Dye-sensitized solar cells based on the principles and materials of photosynthesis: Mechanisms of suppression and enhancement of photocurrent and conversion efficiency. Int. J. Mol. Sci. 2009, 10, 4575-4622.

8. Wang, X.-F.; Koyama, Y.; Nagae, H.; Yamano, Y.; Ito, M.; Wada, Y. Photocurrents of solar cells sensitized by aggregate-forming polyenes: Enhancement due to suppression of singlet-triplet annihilation by lowering of dye concentration or light intensity. Chem. Phys. Lett. 2006, 420, 309-315.

9. Wang, X.-F.; Koyama, Y.; Wada, Y.; Sasaki, S.-i.; Tamiaki, H. A dye-sensitized solar cell using pheophytin-carotenoid adduct: Enhancement of photocurrent by electron and singlet-energy transfer and by suppression of singlet-triplet annihilation due to the presence of the carotenoid moiety. Chem. Phys. Lett. 2007, 439, 115-120.

10. Wang, X.-F.; Zhan, C.-H.; Maoka, T.; Wada, Y.; Koyama, Y. Fabrication of dye-sensitized solar cells using chlorophylls $c_{1}$ and $c_{2}$ and their oxidized forms $c_{1}$ ' and $c_{2}$ ' from Undaria pinnatifida (Wakame). Chem. Phys. Lett. 2007, 447, 79-85.

(C) 2012 by the authors; licensee MDPI, Basel, Switzerland. This article is an open access article distributed under the terms and conditions of the Creative Commons Attribution license (http://creativecommons.org/licenses/by/3.0/). 